\title{
Phase-plane geometries in coupled enzyme assays
}

\author{
Justin Eilertsen $^{\mathrm{a}}$, Wylie Stroberg ${ }^{\mathrm{a}}$, Santiago Schnella,b,c,1 \\ ${ }^{a}$ Department of Molecular $\& 3$ Integrative Physiology, University of Michigan Medical \\ School, Ann Arbor, MI 48109, USA \\ ${ }^{b}$ Department of Computational Medicine $\&$ Bioinformatics, University of Michigan \\ Medical School, Ann Arbor, MI 48109, USA \\ ${ }^{c}$ Brehm Center for Diabetes Research, University of Michigan Medical School, Ann \\ Arbor, MI 48105, USA
}

\begin{abstract}
The determination of a substrate or enzyme activity by coupling of one enzymatic reaction with another easily detectable (indicator) reaction is a common practice in the biochemical sciences. Usually, the kinetics of enzyme reactions is simplified with singular perturbation analysis to derive rate or time course expressions valid under the quasi-steady-state and reactant stationary state assumptions. In this paper, the dynamical behavior of coupled enzyme catalyzed assays is studied by analysis in the phase plane. We analyze two types of time-dependent slow manifolds - Sisyphus and Laelaps manifolds - that occur in asymptotically autonomous vector fields that arise from enzyme coupled assays. Projection onto slow manifolds yields various reduced models, and we develop a mathematical framework from which to analyze coupled enzyme assays through scaling and phase-plane analysis. We demonstrate the necessity of fast indicator reactions to derive mathematical expressions which could be used for the accurate estimation of enzyme kinetic parameters through experimental assays in the laboratory.
\end{abstract}

Keywords: Enzyme kinetics, coupled enzyme assays, Michaelis-Menten reactions, time-dependent slow manifold, Sisyphus manifold, Laelaps manifold, differential-algebraic equation, asymptotically autonomous vector field

Email address: schnells@umich.edu (Santiago Schnell)

${ }^{1}$ Corresponding author 


\section{Introduction}

Biochemical reactions inside cells are generally catalyzed by enzymes, which accelerate the conversion of substrates into products under physiological conditions. Most of the complex chemical processes occurring inside cells or organisms that are necessary for the maintenance of life are catalyzed by enzymes. Consequently, the experimental measurement of enzyme activity through in vitro assays has been substantial importance in biological and biomedical sciences to understand the dynamic of biochemical processes inside cells [1]. Unfortunately, many interesting biochemical processes or macromolecules cannot be observed directly. In the biological science there are many tools - enzymatic or non-enzymatic - to observe indirectly molecular and biological activity. Reporter genes are an excellent example of molecular and non-enzymatic indirect observation of activity; these are genes that enable the detection or measure of gene expression by attaching them to the regulatory sequence of another gene of interest.

Often, the activity of numerous enzymes cannot be observed experimentally through direct observation of their reaction. Instead, these enzyme catalyzed reactions must be observed indirectly by coupling it to another enzyme catalyzed reaction that is used to indicate the non-observable reaction. The observable enzyme catalyzed reaction is known as the indicator or monitor reaction [2]. The non-observable enzyme catalyzed reaction can be of varying complexity; it could follow a linear inhibition or exhibit enzyme degradation [3]. Regardless of the nature of the non-observable reaction, there are two general mechanisms employed to couple enzyme reactions: the sequential enzyme reaction mechanism [4] and the auxiliary enzyme reaction mechanism [5].

To set the stage, and explain how the coupling mechanisms between a nonobservable and an indicator reaction operate, let us assume (for simplicity) that the non-observable reaction follows the Michaelis-Menten (MM) singleenzyme, single-substrate mechanism of action [6]

$$
E_{1}+S_{1} \underset{k_{-1}}{\stackrel{k_{1}}{\rightleftharpoons}} C_{1} \stackrel{k_{2}}{\rightarrow} E_{1}+P
$$

from which, we need to indirectly measure the activity of the enzyme $E_{1}$, through means of an indicator reaction $\left(S_{1}\right.$ is the substrate of the nonobservable reaction, $C_{1}$ the complex, $P$ is the product generated in the 
non-observable reaction). In the sequential enzyme reaction mechanism, the product of the non-observable reaction is the substrate, $S_{2}$ of the indicator reaction. The indicator reaction occurs when $S_{2}$ binds to the sequential indicator enzyme, $E_{2}$, and generates a final product, $P$ :

$$
E_{2}+S_{2} \underset{k_{-3}}{\stackrel{k_{3}}{\rightleftharpoons}} C_{2} \stackrel{k_{4}}{\rightarrow} E_{2}+P .
$$

In the above mechanism, $k_{1}, k_{1}, k_{3}, k_{-3}$ are microscopic rate constants and $k_{2}, k_{4}$ are catalytic constants. $C_{1}$ and $C_{2}$ are intermediate enzyme-substrate complexes. The sequential assay is by far the most common type of coupled assay, and there are many examples reported in the literature (see, Tables II and III in [3] and Table 4.5 in [2]). One example is the phosphorylation of glucose to glucose- 6 phosphate requires energy and so it is coupled to the hydrolysis of ATP to ADP and Pi. The primary reaction is catalyzed by hexokinase, and is non-observable in typical steady-state kinetic experiments. Therefore, to investigate the hexokinase activity, its reaction is coupled to the catalytic conversion of glucose-6 phosphate into 6-P gluconolactone with the enzyme glucose 6-P dehydrogenase. The later serves as an indicator reaction.

A less common coupled assay is the auxiliary assay. In the auxiliary enzyme reaction mechanisms, the product of the non-observable reaction is the indicator enzyme $E_{2}$, which binds with the substrate $S_{2}$ to form a product:

$$
\begin{aligned}
E_{1}+S_{1} \underset{k_{-1}}{\rightleftharpoons} C_{1} \stackrel{k_{2}}{\rightarrow} E_{1}+E_{2} \\
E_{2}+S_{2} \underset{k_{-3}}{\stackrel{k_{3}}{\rightleftharpoons}} C_{2} \stackrel{k_{4}}{\rightarrow} E_{2}+P .
\end{aligned}
$$

The physiologic response to a vascular lesion entails a number of enzymatic steps, which leads to clot formation. These enzymatic steps are a cascade of enzyme catalyzed reactions following the auxiliary enzyme mechanism described above [7]. In the laboratory, the activity of thrombin, one of the blood coagulation enzymes, has been studied with a coupled auxiliary enzyme assay. Thrombin catalyzes the activation of protein $\mathrm{P}$, which is non-observable 
using steady-state kinetic laboratory assays. However, the formation of pnitroaniline from substrate S2266 is catalyzed by activated protein P, and is observable through steady-state kinetic progress curve experiments. By coupling the two reactions, thrombin function is studied with a coupled auxiliary enzyme assay [8].

The MM equation is usually employed to measure the enzyme kinetics of the primary reaction when it can be observed experimentally in steady-state experiments. The MM equation itself is given by

$$
\frac{d p}{d t}=\frac{V_{1} s_{1}}{K_{M_{1}}+s_{1}},
$$

where $V_{1}$ is the limiting rate of the primary reaction and $K_{M_{1}}$ is its Michaelis constant $[9,10]$

$$
K_{M_{1}}=\frac{k_{-1}+k_{2}}{k_{1}} .
$$

The Michaelis constant is defined operationally as the concentration of the substrate at which the rate of the reaction is half of the limiting rate; that is, $\mathrm{d} p / \mathrm{d} t=0.5 V_{1}$. The enzyme specificity is characterized through the specificity constant, which is the result of dividing $k_{2}$ by $K_{M_{1}}[11,12,13]$.

The kinetic constants $V_{1}$ and $K_{M_{1}}$ are generally estimated through initial rate or time course experiments $[14,2]$ by mathematically solving an inverse problem $[15,16]$. In the case of coupled enzyme assays, the caveat with this procedure is that the primary reaction is not experimentally observable. Thus, in coupled enzyme assays, $V_{1}$ and $K_{M_{1}}$ need to be estimated through means of indirect measures of data recorded from the indicator reaction. From a theoretical point of view the demand is obvious; a mathematical theory must be developed that is capable of accurately describing the relationship between the non-observable reaction and the indicator reaction. To date, a nonlinear theory has not been established, even though coupled enzyme catalyzed reactions are commonly used to study the kinetics of non-observable reactions. Most enzyme kinetic analyses developed to study coupled enzyme assays assume that the coupled enzyme reactions follow first-order kinetics $[17,18,19,20,21]$, and limit to measure the lag time of the reaction, which is effectively the length of time it takes before measurable formation rates of $P$ become experimentally detectable.

Accordingly, one of the main contributions of this paper is the development of utilizable mathematical techniques to investigate the kinetics of 
enzyme catalyzed indicator reactions in typical state-state laboratory assays. To construct a mathematical framework of indicator equations, we observe that, from the vantage point of a mathematical landscape, the validity of the MM equation resides in the presence of disparate timescales (slow and fast timescales) inherent in the non-observable reaction. The separation of these timescales is known as the Quasi-Steady-State Approximation (QSSA). In short, intrinsic slow and fast timescales within the non-observable reaction will imply the existence of a one-dimensional slow manifold on which the MM equation is a valid approximation to the two-dimensional mass action kinetic model [22]. Because the indicator reaction is enzyme catalyzed, it will also exhibit (under certain circumstances) slow and fast timescales. Thus, model reduction methods based on slow manifold projections will be central, although not always necessary, in the analysis and model reduction of the indicator reaction. Central to the slow manifold projection method is the presence of quasi-steady-state (QSS) dynamics, which are generally assumed to hold whenever substrate concentrations are in excess of enzyme concentrations. "Test tube" experiments can be designed so that concentrations are favorable for QSS dynamics. Although the current experimental technology does not allow the reliable estimation of enzyme kinetic parameters intracellularly yet, the QSS dynamics can hold inside cells as the concentration of most substrates exceed their cognature enzyme concentrations in several tissues and organisms (see, [23] for a meta-analysis).

From a theorists' perspective, the dynamics of the indicator reaction are mathematically interesting. As we will show, the vector fields that determine the transient kinetics of the indicator reaction are asymptotically autonomous. The novelty of asymptotically autonomous vector fields is that, since they are a unique class of non-autonomous vector fields, the slow manifolds that are present in them will naturally be time-dependent. This is in contrast to the stationary slow manifold in the single enzyme, single substrate reaction when the QSSA or reactant stationary assumption (RSA) holds $[24,25]$. Moreover, the existence of slow manifolds in the asymptotically autonomous vector fields is dependent on the rate kinetics of the non-observable reaction, and physical initial conditions restrict the initial velocities of the indicator reaction to be identically zero. As a result, it is tempting to assume that indicator reactions, projected onto a slow manifold, will lack a "fast transient" since experimental initial conditions would suggest that phase-plane trajectories circumnavigate the fast temporal boundary layer. We will explore, from both the mathematical and biochemical moti- 
vation, the influence of fast (short) timescales that naturally arise from the scaling analysis of the indicator reaction. In addition, we will show that, in the limiting case of an extremely fast sequential enzyme reaction, the phaseplane dynamics can be projected onto a zero-dimensional manifold. This analysis demonstrates the necessity of fast indicator reactions in coupled assays. While such assumptions have previously been endorsed, it has never been established as a mathematical result from nonlinear analysis. This is a novel result in the context of coupled enzyme kinetics, and serves as the primary contribution of this paper.

\section{Mathematical background of critical manifolds for dynamical systems with fast and slow scales}

Here we briefly review the necessary mathematical background that is pertinent to fast/slow systems and slow manifolds. If we consider a system of the form

$$
\begin{aligned}
\dot{x} & =f(x, y), \quad x \in \mathbb{R}^{n}, \quad f: \mathbb{R}^{n} \times \mathbb{R}^{m} \rightarrow \mathbb{R}^{n}, \\
\varepsilon \dot{y} & =g(x, y), \quad y \in \mathbb{R}^{m}, \quad g: \mathbb{R}^{m} \times \mathbb{R}^{n} \rightarrow \mathbb{R}^{m},
\end{aligned}
$$

where $\varepsilon$ is a constant such that $\varepsilon \ll 1$, then as a result of Tikhonov and Fenichel we have the following theorem [26]:

Theorem 1. Let $\mathcal{M}$ be a compact and normally hyperbolic manifold of the critical manifold $\mathcal{M}_{0}$ and let $f, g$ of ( $7 a$ ) and (7b) have $r$ continuous derivatives (i.e., $f, g \in C^{r}$ with $\left.r<\infty\right)$ ). If $0<\varepsilon \ll 1$ then the following are true:

(1) $\exists$ a locally invariant manifold $\mathcal{M}_{\varepsilon}$ that is diffeomorphic to $\mathcal{M}_{0}$.

(2) The Hausdorff distance from $\mathcal{M}_{\varepsilon}$ to $\mathcal{M}_{0}$ is $\mathcal{O}(\varepsilon)$

(3) The flow in $\mathcal{M}_{\varepsilon}$ converges to flow on $\mathcal{M}_{0}$ as $\varepsilon \rightarrow 0$.

(4) $\mathcal{M}_{\varepsilon}$ is a $C^{r}$-differentiable manifold.

(5) The stability of $\mathcal{M}_{\varepsilon}$ is the same as the stability of $\mathcal{M}_{0}$.

(6) $\mathcal{M}_{\varepsilon}$ may not be unique, but all admissible manifolds that satisfy (1)-(5) will be at distance apart that is $\mathcal{O}\left(e^{-\omega / \varepsilon}\right)$ (with $\left.0<\omega<\infty\right)$. 
The critical manifold $\mathcal{M}_{0}$ of Theorem 1 refers to the zero level set of $g(x, y)$ :

$$
\mathcal{M}_{0} \equiv\{(x, y): g(x, y)=0\} .
$$

If we solve (8) and obtain an expression $y=h(x)$ with $g(x, h(x))=0$, then the stability properties of the critical manifold can be determined by the location of the eigenvalues of the matrix $J_{\mathcal{M}_{0}}$

$$
J_{\mathcal{M}_{0}}=D g(x, h(x)),
$$

where " $D$ " is used to denote the derivative with respect to the fast variables " $y$ ". If the eigenvalues of $J_{\mathcal{M}_{0}}$ have negative real parts, then $\mathcal{M}_{0}$ is attracting and hence $\mathcal{M}_{\varepsilon}$ will inherit the same attribute. In addition to conditions that imply the presence of a slow manifold, we obtain from the Fenichel-Tikhonov theorem the leading order approximation to (7a) and (7b):

$$
\begin{aligned}
\dot{x} & =f(x, h(x))+\mathcal{O}(\varepsilon) \\
y & =h(x)+\mathcal{O}(\varepsilon) .
\end{aligned}
$$

The validity of the leading order approximation (10b) in the context of asymptotically autonomous vector fields will be a subject of critical importance in the upcoming sections. Formally, an asymptotically autonomous vector field consists of a vector field,

$$
f(x, t) \quad x \in \mathbb{R}^{n}
$$

such that

$$
\lim _{t \rightarrow \infty} f(x, t)=g(x) .
$$

Thus, an asymptotically autonomous vector field is a vector field that becomes autonomous in the limiting case as $t \rightarrow \infty[27]$.

\section{Analysis of the sequential enzyme reaction mechanism}

We start our analysis with the sequential enzyme reaction mechanism represented by the chemical equations (1)-(2), which consists of a singlesubstrate, single-enzyme non-observable reaction followed by another singlesubstrate, single-enzyme observable reaction (indicator reaction). In this mechanism, the product of the non-observable reaction becomes the substrate of the indicator reaction. By applying the law of mass action to (1)-(2), we obtain a nonlinear system of differential equations with three conservation laws [5]. We begin our analysis by scaling the mass action equations. 


\subsection{Scaling of the sequential enzyme reaction}

After eliminating redundant expressions using the conserved quantities $s_{1}^{0}, e_{1}^{0}$, and $e_{2}^{0}$, the mass action equations that model the sequential enzyme mechanism are:

$$
\begin{aligned}
& \dot{s}_{1}=-k_{1}\left(e_{1}^{0}-c_{1}\right) s_{1}+k_{-1} c_{1} \\
& \dot{c}_{1}=k_{1}\left(e_{1}^{0}-c_{1}\right) s_{1}-\left(k_{-1}+k_{2}\right) c_{1} \\
& \dot{s}_{2}=-k_{3}\left(e_{2}^{0}-c_{2}\right) s_{2}+k_{-3} c_{2}+k_{2} c_{1} \\
& \dot{c}_{2}=k_{3}\left(e_{2}^{0}-c_{2}\right) s_{2}-\left(k_{-3}+k_{4}\right) c_{2} .
\end{aligned}
$$

The lowercase letters $s_{1}, c_{1}, e_{1}$ and $s_{2}, c_{2}, e_{2}, p$ denote the concentrations of $S_{1}, C_{1}, E_{1}$ and $S_{2}, C_{2}, E_{2}, P$ respectively. Notice equations (13a)-(13b) are autonomous and independent of $s_{2}$ and $c_{2}$. In this regard, the first catalyzed reaction drives the second catalyzed reaction; thus, the indicator reaction can be viewed as a non-autonomous system with forcing term $k_{2} c_{1}(t)$.

The complete catalyzed sequential enzyme reaction (13) is characterized by four timescales $t_{c_{1}}, t_{s_{1}}, t_{c_{2}}$ and $t_{s_{2}}$, which are easily derived by the method of Rice [28, 29],

$$
\begin{array}{ll}
t_{c_{1}}=\frac{1}{k_{1}\left(K_{M_{1}}+s_{1}^{0}\right)}, & t_{s_{1}}=\frac{K_{M_{1}}+s_{1}^{0}}{k_{2} e_{1}^{0}} \\
t_{c_{2}}=\frac{1}{k_{3}\left(K_{M_{2}}+s_{1}^{0}\right)}, & t_{s_{2}}=\frac{K_{M_{2}}+s_{1}^{0}}{k_{4} e_{2}^{0}}
\end{array}
$$

with $K_{M_{1}}$ and $K_{M_{2}}$ denoting the Michaelis constants:

$$
K_{M_{1}}=\frac{k_{-1}+k_{2}}{k_{1}} \quad K_{M_{2}}=\frac{k_{-3}+k_{4}}{k_{3}} .
$$

The timescales $t_{c_{1}}$ and $t_{s_{1}}$ define, respectively, the temporal order of magnitude of the initial fast transient and the approximate length of non-observable reaction [30]. Analogously, the timescale $t_{c_{2}}$ is a fast timescale, and $t_{s_{2}}$ is the approximate time it takes the indicator reaction to complete. Thus, $t_{c_{1}}$ and $t_{c_{2}}$ are fast timescales, while $t_{s_{1}}$ and $t_{s_{2}}$ are slow timescales.

To establish the presence of slow manifolds, we rescale the mass action 
equations with respect to the following dimensionless variables

$$
\begin{aligned}
& T=\frac{t}{t_{s_{1}}} \quad \bar{s}_{1}=\frac{s_{1}}{s_{1}^{0}} \quad \bar{c}_{1}=\frac{\left(K_{M_{1}}+s_{1}^{0}\right)}{e_{1}^{0} s_{1}^{0}} c_{1}, \\
& \tau=\frac{t}{t_{s_{2}}}, \quad \bar{s}_{2}=\frac{s_{2}}{s_{2}^{\max }}, \quad \bar{c}_{2}=\frac{\left(K_{M_{2}}+s_{2}^{\max }\right)}{e_{2}^{0} s_{2}^{\max }} c_{2},
\end{aligned}
$$

where $s_{2}^{\max }$ denotes the maximum amount of unbound indicator substrate observed in the reaction. In dimensionless form, the mass action equations (13) that govern the non-observable reaction are:

$$
\begin{gathered}
\frac{d \bar{s}_{1}}{d T}=\left(1+\sigma_{1}\right)\left(1+\kappa_{1}\right)\left[\left(\frac{\sigma_{1}}{1+\sigma_{1}} \bar{c}_{1}-1\right) \bar{s}_{1}+\frac{\alpha_{1}}{1+\sigma_{1}} \bar{c}_{1}\right], \\
\varepsilon \frac{d \bar{c}_{1}}{d T}=\left(1+\sigma_{1}\right)\left(1+\kappa_{1}\right)\left[\left(1-\frac{\sigma_{1}}{1+\sigma_{1}} \bar{c}_{1}\right) \bar{s}_{1}-\frac{1}{1+\sigma_{1}} \bar{c}_{1}\right] .
\end{gathered}
$$

The dimensionless equations that describe the indicator reaction are:

$$
\begin{aligned}
\frac{d \bar{s}_{2}}{d \tau} & =\left(1+\sigma_{2}\right)\left(1+\kappa_{2}\right)\left[\left(\frac{\tilde{\sigma}_{2}}{1+\tilde{\sigma}_{2}} \bar{c}_{2}-1\right) \bar{s}_{2}+\frac{\alpha_{2}}{1+\tilde{\sigma}_{2}} \bar{c}_{2}\right]+\Lambda \delta_{S} \bar{c}_{1}, \\
\lambda \frac{d \bar{c}_{2}}{d \tau} & =\left(1+\tilde{\sigma}_{2}\right)\left(1+\kappa_{2}\right)\left[\left(1-\frac{\tilde{\sigma}_{2}}{1+\tilde{\sigma}_{2}} \bar{c}_{2}\right) \bar{s}_{2}-\frac{1}{1+\tilde{\sigma}_{2}} \bar{c}_{2}\right] .
\end{aligned}
$$

The variables $\tilde{\sigma}_{2}, \sigma_{1}, \sigma_{2}, \kappa_{1}$, and $\kappa_{2}$ given by,

$$
\tilde{\sigma}_{2} \equiv s_{2}^{\max } / K_{M_{2}}, \quad \sigma_{1} \equiv s_{1}^{0} / K_{M_{1}}, \quad \sigma_{2} \equiv s_{1}^{0} / K_{M_{2}}, \quad \kappa_{1} \equiv k_{-1} / k_{2}, \quad \kappa_{2} \equiv k_{-3} / k_{4}
$$

and the constants $\alpha_{1}$ and $\alpha_{2}$ are dependent on $\kappa_{1}$ and $\kappa_{2}$ respectively,

$$
\alpha_{1}=\kappa_{1} /\left(1+\kappa_{1}\right), \quad \alpha_{2}=\kappa_{2} /\left(1+\kappa_{2}\right) .
$$

The constants $\varepsilon$ and $\lambda$ are dependent on the initial enzyme and substrate concentrations and the Michaelis constants

$$
\varepsilon=\frac{e_{1}^{0}}{K_{M_{1}}+s_{1}^{0}}, \quad \lambda=\frac{e_{2}^{0}}{K_{M_{2}}+s_{1}^{0}},
$$

and the ratios $\Lambda$ and $\delta_{S}$ are:

$$
\Lambda=\frac{s_{1}^{0}}{s_{2}^{\max }}, \quad \delta_{S}=\frac{t_{s_{2}}}{t_{s_{1}}} .
$$


Scaling the indicator reaction with respect to $\tau=t / t_{s_{2}}$ is no accident. This is because, if the indicator reaction is slow, then $t_{s_{2}}$ gives a very good estimate of the completion timescale corresponding to the indicator reaction. Moreover, the ratio $\delta_{S}$ should give a good indication of how well the indicator reaction "keeps up" with the non-observable reaction. Since the completion of the indicator reaction cannot occur before the completion of the nonobservable reaction, we would expect that if $\delta_{S} \ll 1$, then the completion of the indicator reaction will occur at roughly the same time as the nonobservable reaction.

The ratio, $\Lambda$, will be very large if the indicator reaction is fast; this is because for fast indicator reactions, $s_{2}$ should quickly bind with $e_{2}$ and form product. Consequently, the maximum amount of unbound indicator substrate $s_{2}$ should be much less than the initial non-observable susbtrate $s_{1}^{0}$. In contrast, if the indicator reaction is slow in comparison to the nonobservable reaction (i.e., if $t_{s_{2}} \gg t_{s_{1}}$ ), then $\Lambda \approx 1$.

If $\varepsilon, \lambda \ll 1$, then there will exists slow manifolds $\mathcal{M}_{\varepsilon}, \mathcal{M}_{\lambda}$, whose leading order expansions are:

$$
\begin{aligned}
& \mathcal{M}_{\varepsilon}=\mathcal{M}_{0}+\mathcal{O}(\varepsilon) \quad \text { with } \quad \mathcal{M}_{0} \equiv c_{1}-\frac{e_{1}^{0}}{K_{M_{1}}+s_{1}} s_{1}=0 \\
& \mathcal{M}_{\lambda}^{t}=\mathcal{M}_{0, \lambda}+\mathcal{O}(\lambda) \quad \text { with } \quad \mathcal{M}_{0, \lambda} \equiv c_{2}-\frac{e_{2}^{0}}{K_{M_{2}}+s_{2}} s_{2}=0
\end{aligned}
$$

The presence of the slow manifolds, $\mathcal{M}_{\lambda}$ and $\mathcal{M}_{\varepsilon}$, implies that

$$
\begin{aligned}
& \dot{s}_{1}=-\frac{V_{1}}{K_{M_{1}}+s_{1}} s_{1}+\mathcal{O}(\varepsilon) \\
& \dot{s}_{2}=-\frac{V_{2}}{K_{M_{2}}+s_{2}} s_{2}+k_{2} c_{1}+\mathcal{O}(\lambda)
\end{aligned}
$$

are good zeroth order approximations to the mass action equations on the $T$ and $\tau$ timescales, respectively. Moreover, after the initial fast transient of the non-observable reaction, equation (24b) can be reduced to

$$
\dot{s}_{2}=-\frac{V_{2}}{K_{M_{2}}+s_{2}} s_{2}+\frac{V_{1}}{K_{M_{1}}+s_{1}} s_{1}+\mathcal{O}(\lambda)+\mathcal{O}(\varepsilon) .
$$

The validity of (23a) is well-established and, we will not go into the details of this here. What is interesting in this case is the criteria for the validity of 
(23b). While the qualifier " $\lambda \ll 1$ " is sufficient to establish the presence of a slow manifold, and scaling analysis can give us an idea of just how small $\lambda$ should be in order for first order approximations to be "good" on the depletion timescale, it not clear if the first order approximation is good on shorter timescales (i.e., does the QSSA remain valid on timescales that are shorter than $t_{s_{2}}$ ?).

Several remarks need to be made before we begin to establish conditions for the first order validity of (23b). First, although $\mathcal{M}_{\lambda}$ is a time-dependent manifold, its explicit dependence on time is determined by the $\mathcal{O}(\lambda)$ terms. Consequently, the manifold will evolve in the two-dimensional phase-plane, and the slow and fast timescales of both the indicator and non-observable reactions will play an integral role in understanding the validity of the first order truncation. Second, because experimental initial conditions typically lie on the $c_{2}$-nullcline, it's not immediately clear if we should expect to see an initial fast transient towards the slow manifold over some fast timescale since the solution is on the $c_{2}$-nullcline is already when $t=0$. Thus, two questions arise that must be answered: (1) how small should $\lambda$ be in order to ensure a first order truncation is valid, and what does scaling the mass-action equations with respect to shorter (long timescales) say about the validity of the QSSA? (2) If the timescale $t_{c_{2}}$ does not account for a fast response under typical experimental conditions, then what, if anything, does it account for? We begin with a qualitative description of $\mathcal{M}_{\lambda}$ in order to answer these questions.

\subsection{The sequential enzyme reaction exhibits a Sisyphus manifold}

When the indicator reaction is roughly the same speed as the non-observable reaction (i.e., $\delta_{S} \approx 1$ ), and $\lambda \ll 1$, then the phase plane of the indicator reaction exhibits what we call a Sisyphus manifold. In this case, $s_{2}^{\max }$ will be proportional in magnitude (although still less than) $s_{1}^{0}$. Thus, we do not expect $\Lambda$ to be very small. Consequently, it is permissible to scale $s_{2}$ with respect to $s_{1}^{0}$ when $\delta_{S} \approx 1$. Scaling the mass action equations with respect to the dimensionless time $\tau$, and $s_{2}^{\max } \equiv s_{1}^{0}$ yields:

$$
\begin{aligned}
\frac{d \bar{s}_{2}}{d \tau} & =\left(1+\sigma_{2}\right)\left(1+\kappa_{2}\right)\left[\left(\frac{\sigma_{2}}{1+\sigma_{2}} \bar{c}_{2}-1\right) \bar{s}_{2}+\frac{\alpha_{2}}{1+\sigma_{2}} \bar{c}_{2}\right]+\delta_{S} \bar{c}_{1}, \\
\lambda \frac{d \bar{c}_{2}}{d \tau} & =\left(1+\sigma_{2}\right)\left(1+\kappa_{2}\right)\left[\left(1-\frac{\sigma_{2}}{1+\sigma_{2}} \bar{c}_{2}\right) \bar{s}_{2}-\frac{1}{1+\sigma_{2}} \bar{c}_{2}\right] .
\end{aligned}
$$


If $\lambda \ll 1$, then at zeroth order, for $t \geq t_{c_{1}}$, we have:

$$
\begin{aligned}
& \dot{s}_{2}=-\frac{V_{2}}{K_{M_{2}}+s_{2}} s_{2}+\frac{V_{1}}{K_{M_{1}}+s_{1}} s_{1}, \\
& c_{2}=\frac{e_{2}^{0}}{K_{M_{2}}+s_{2}} s_{2} .
\end{aligned}
$$

The solution to $(27 \mathrm{a})-(27 \mathrm{~b})$, which starts on the $c_{2}$-nullcline when experimental initial conditions are prescribed, essentially moves up and down the $c_{2}$-nullcline. Since the $c_{2}$-nullcline resembles a hill, we refer to the slow manifold, $\mathcal{M}_{\lambda}$, that lies close to the $c_{2}$-nullcline, as the Sisyphus manifold, after the Greek mythological king who was sentenced for eternity to push a stone up a hill only to have it roll back down as it neared the top (see FigurE 1 and Movie 1 in the Supplementary Material).

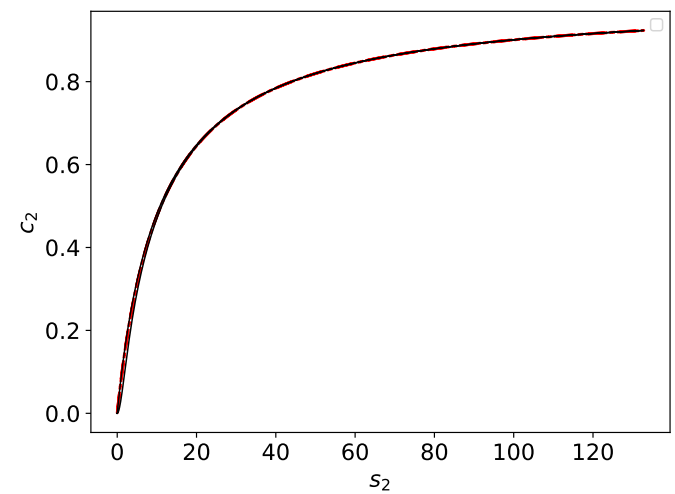

Figure 1: The numerical solution of the mass action equations (13) (thin black curve) moves up, then down, the $c_{2}$-nullcline in phase-space for the sequential enzyme reaction mechanism. Movement is illustrated dynamically in Movie 1 available in the Supplementary Material. The dimensionless units using in the numerical integration are: $s_{1}^{0}=1000, e_{1}^{0}=1, e_{2}^{0}=1, k_{1}=1, k_{2}=10, k_{-3}=1, k_{3}=1, k_{4}=10, k_{-3}=1$.

We invoke moving nullcline analysis to geometrically illustrate why solutions roll up and then slide down the $c_{2}$-nullcline. Starting with some basic notation, we will denote the respective $s_{2}$ and $c_{2}$ nullclines as

$$
\begin{aligned}
& \left\{\left(s_{2}, c_{2}\right) \in \mathbb{R}^{2} \mid c_{2}-\frac{k_{3} e_{2}^{0} s_{2}-k_{2} c_{1}}{k_{3} s_{2}+k_{-3}}=0\right\} \equiv \mathcal{N}_{s_{2}}^{t}, \\
& \left\{\left(s_{2}, c_{2}\right) \in \mathbb{R}^{2} \mid c_{2}-\frac{e_{2}^{0}}{K_{M_{2}}+s_{2}} s_{2}=0\right\} \equiv \mathcal{N}_{c_{2}},
\end{aligned}
$$


where the superscript " $t$ " in (28a) denotes the time-dependency of the $s_{2^{-}}$ nullcline. If we consider snapshots of the $s_{2}-c_{2}$ phase plane at different points in time (i.e., let $t=t_{n}$ ), we see that there is a fixed point, $\boldsymbol{x}^{*}\left(t_{n}\right)$,

$$
\boldsymbol{x}^{*}\left(t_{n}\right)=\mathcal{N}_{c_{2}} \bigcap \mathcal{N}_{s_{2}}^{t=t_{n}}
$$

that slides, like a bead on a wire, up and down the $c_{2}$-nullcline. Algebraically, the fixed point $\boldsymbol{x}^{*}(t)$ is given by

$$
s_{2}=\frac{K_{M_{2}}}{V_{2}-k_{2} c_{1}} k_{2} c_{1}, \quad c_{2}=\frac{e_{2}^{0}}{K_{M_{2}}+s_{2}} s_{2} .
$$

An interesting observation can be made here. As $V_{2} \rightarrow k_{2} c_{1}$, the maximum distance from $\boldsymbol{x}^{*}$ to the origin becomes arbitrarily large:

$$
\lim _{V_{2} \rightarrow k_{2} c_{1}}\left(\frac{K_{M_{2}}}{V_{2}-k_{2} c_{1}} k_{2} c_{1}\right)=\infty, \quad \lim _{s_{2} \rightarrow \infty}\left(\frac{e_{2}^{0}}{K_{M_{2}}+s_{2}} s_{2}\right)=e_{2}^{0} .
$$

In contrast, as $V_{2}$ gets arbitrarily large, the maximum distance from $\boldsymbol{x}^{*}$ to the origin gets negligibly small:

$$
\lim _{V_{2} \rightarrow \infty}\left(\frac{K_{M_{2}}}{V_{2}-k_{2} c_{1}} k_{2} c_{1}\right)=0, \quad \lim _{s_{2} \rightarrow 0}\left(\frac{e_{2}^{0}}{K_{M_{2}}+s_{2}} s_{2}\right)=0 .
$$

What phase-space trajectories do is follow the sliding fixed point and, if $\lambda$ is sufficiently small, the phase plane trajectory will follow the fixed point along a path that is extremely close $c_{2}$-nullcline. This typically occurs in three stages: (1) the trajectory chases the fixed point up the $c_{2}$-nullcline, (2) the trajectory "catches" the fixed point, at which time both $s_{2}$ and $c_{2}$ reach their maximum values and, (3) the trajectory follows the fixed point back down the $c_{2}$-nullcline (see FiguRES 2a- $2 \mathrm{~d}$ for another visualization of the Sisyphus manifold). The speed of the indicator reaction determines how far the fixed point, $\boldsymbol{x}^{*}$, can travel away from the origin. For fast indicator reactions, we see that the maximum distance from $\boldsymbol{x}^{*}$ to the origin to be very small, and thus we expect that $s_{2}^{\max }$ will be very small. In contrast, the fixed point will travel very far away from the origin if the indicator reaction is slow, and thus we expect $s_{2}^{\max } \approx s_{1}^{0}$. 


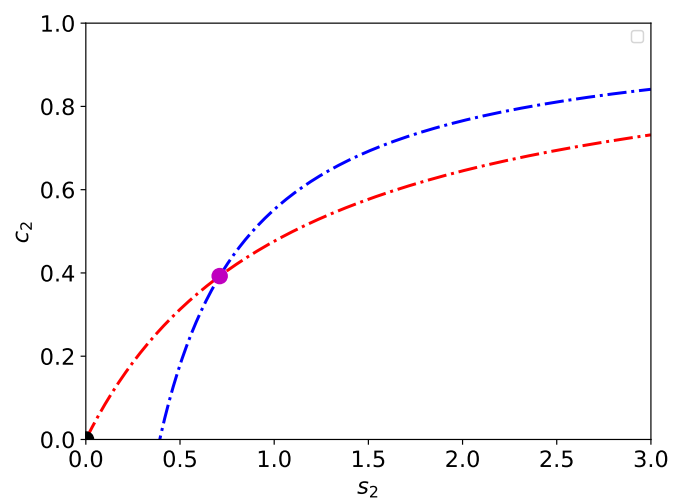

(a)

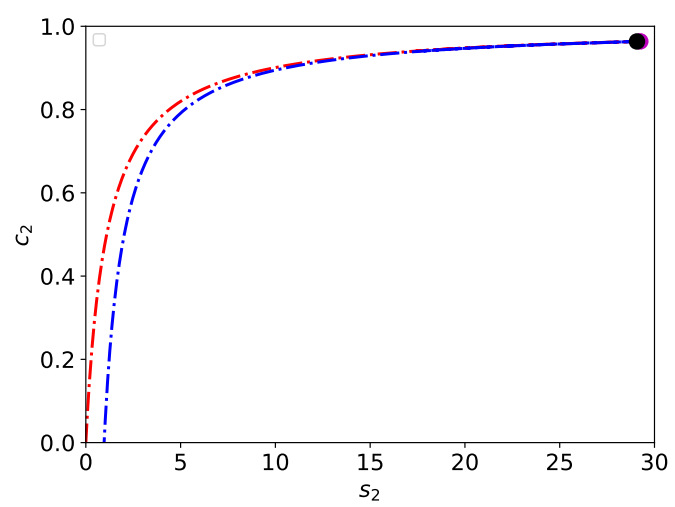

(c)

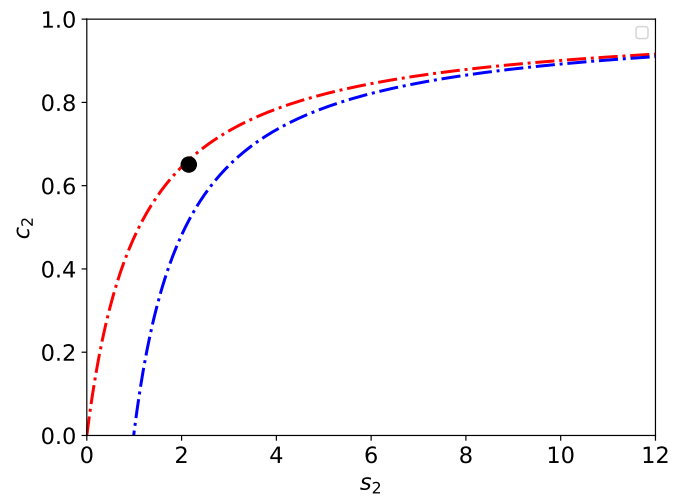

(b)

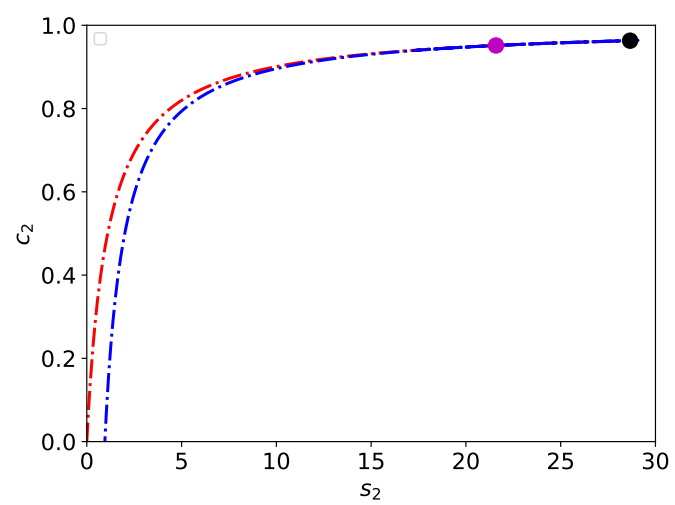

(d)

Figure 2: Visualization of the Sisyphus manifold in the sequential enzyme reaction mechanism. The numerical solution of (13) (black dot) follows the intersection, $\boldsymbol{x}^{*}$ (purple dot) of the nullclines, along a path that can be approximated by the $c_{2}$-nullcline (panels (a) and (b)). Eventually, the solution catches $\boldsymbol{x}^{*}$ (panel (c)) and then chases $\boldsymbol{x}^{*}$ back down the $c_{2}$-nullcline (d). In the panels, the initial conditions and parameter values are: $e_{1}^{0}=1, s_{1}^{0}=1000, k_{1}=1, k_{2}=10$ and $k_{-1}=1 . s_{2}^{0}=0, e_{2}^{0}=1, k_{3}=10, k_{4}=10$ and $k_{-3}=1$.

\subsection{Analysis of slow and fast sequential indicator reactions}

As we saw in the previous section, when $t_{s_{2}} \approx t_{s_{1}}$, the indicator reaction is effectively in a QSS for (seemingly) most of the coupled sequential enzyme reaction mechanism. We now want to consider the cases when the indicator is very fast, or very slow, in comparison to the non-observable reaction. It must 
be noted that the completion of the indicator reaction cannot occur before the completion of the non-observable reaction for the coupled sequential enzyme reaction mechanism. Thus, a fast indicator is taken to be synonymous with small maximum displacement of $\boldsymbol{x}^{*}$.

\subsubsection{Analysis of extremely fast indicator reactions}

The first form of the indicator reaction we will consider is the case when $V_{2} \gg V_{1}$, and the indicator reaction is incredibly fast. What phase space trajectories do in the case of the sequential enzyme reaction is chase the moving fixed point $\boldsymbol{x}^{*}$. Given the limits computed in (32), we expect the phase plane trajectory to "catch" $\boldsymbol{x}^{*}$ very quickly when the indicator reaction is fast. This means that (30) will serve as a very good approximation to the mass action equations over measurable timescales. In fact, we can simplify the expression even further in the limiting case. First, we write the expression for $s_{2}$ as

$$
s_{2}=\frac{K_{M_{2}}}{V_{2}-k_{2} c_{1}} k_{2} c_{1}=\frac{K_{M_{2}}}{V_{2}\left(1-\frac{k_{2} c_{1}}{V_{2}}\right)} k_{2} c_{1} .
$$

Next, employing a Taylor series expansion we have

$$
\frac{1}{1-\frac{k_{2} c_{1}}{V_{2}}}=1+\mathcal{O}(\phi), \quad \phi \equiv \max \frac{k_{2} c_{1}}{V_{2}} .
$$

Thus, we can take

$$
s_{2}=K_{M_{2}} \phi+\mathcal{O}\left(\phi^{2}\right)
$$

as a leading order solution to $s_{2}$ when the indicator reaction is fast. Inserting (35) into $\dot{p}=V_{2} s_{2} /\left(K_{M_{2}}+s_{2}\right)$, and taking the limit as $\phi \rightarrow 0$ yields

$$
\dot{p}=\frac{V_{1}}{K_{M_{1}}+s_{1}} s_{1}+\mathcal{O}(\phi),
$$

provided $\varepsilon \ll 1$, and the non-observable reaction is in a QSS for the duration of the reaction. Therefore, the rate expression for the product formation is equivalent to the rate expression for the single-enzyme, single-substrate reaction when the indicator reaction is extremely fast. What is remarkable about this approximation is its condition for validity, namely that,

$$
\max _{t>0} k_{2} c_{1} \ll V_{2} \equiv \frac{V_{2}}{V_{1}} \gg \frac{\sigma_{1}}{1+\sigma_{1}} \text {. }
$$


Notice that is not necessary that $\lambda \ll 1$, and the restriction that $e_{2}^{0}$ be less than $s_{1}^{0}$ is not required. In fact, large concentrations of $e_{2}^{0}$ will actually be beneficial, as the indicator reaction will tend to speed up with increasing initial concentrations of the indicator enzyme $e_{2}$ in the coupled sequential enzyme reaction mechanism.

To put the proverbial "nail in the coffin", we examine how the same approximation can be obtained through scaling. If the indicator reaction is fast, then $\delta_{S} \ll 1$. Additionally, since we can assume, based on our geometrical observations in the phase plane, that $s_{2}^{\max } \ll s_{1}^{0}$, which implies that $\Lambda \gg 1$. Then, we will expect $t_{s_{1}}$ to provide a reasonable depletion timescale when the indicator reaction is fast. Rescaling the mass action equations with respect to $T=t / t_{s_{1}}$,

$$
\begin{aligned}
\frac{d \bar{s}_{2}}{d T} & =\frac{\left(1+\sigma_{2}\right)\left(1+\kappa_{2}\right)}{\delta_{S}}\left[\left(\frac{\tilde{\sigma}_{2}}{1+\tilde{\sigma}_{2}} \bar{c}_{2}-1\right) \bar{s}_{2}+\frac{\alpha_{2}}{1+\tilde{\sigma}_{2}} \bar{c}_{2}\right]+\Lambda \bar{c}_{1} \\
\lambda \frac{d \bar{c}_{2}}{d T} & =\frac{\left(1+\tilde{\sigma}_{2}\right)\left(1+\kappa_{2}\right)}{\delta_{S}}\left[\left(1-\frac{\tilde{\sigma}_{2}}{1+\tilde{\sigma}_{2}} \bar{c}_{2}\right) \bar{s}_{2}-\frac{1}{1+\tilde{\sigma}_{2}} \bar{c}_{2}\right]
\end{aligned}
$$

and multiplying through by $\delta_{S}$ yields

$$
\begin{gathered}
\delta_{S} \frac{d \bar{s}_{2}}{d T}=\left(1+\sigma_{2}\right)\left(1+\kappa_{2}\right)\left[\left(\frac{\tilde{\sigma}_{2}}{1+\tilde{\sigma}_{2}} \bar{c}_{2}-1\right) \bar{s}_{2}+\frac{\alpha_{2}}{1+\tilde{\sigma}_{2}} \bar{c}_{2}\right]+\delta_{S} \Lambda \bar{c}_{1} \\
\delta_{S} \lambda \frac{d \bar{c}_{2}}{d T}=\left(1+\tilde{\sigma}_{2}\right)\left(1+\kappa_{2}\right)\left[\left(1-\frac{\tilde{\sigma}_{2}}{1+\tilde{\sigma}_{2}} \bar{c}_{2}\right) \bar{s}_{2}-\frac{1}{1+\tilde{\sigma}_{2}} \bar{c}_{2}\right] .
\end{gathered}
$$

As $\delta_{S} \rightarrow 0$, both terms on the left hand sides of (39a)-(39b) vanish, and we see that the indicator reaction is reducible through slow manifold projection (keep in mind that as $\delta_{S} \rightarrow 0, \Lambda \rightarrow \infty$, and therefore the term $\delta_{S} \Lambda \bar{c}_{1}$ is not asymptotically negligible). What is unique in the limiting case is that the slow manifold is zero-dimensional (i.e., it reduces to a single fixed point, $\left.\boldsymbol{x}^{*}=(0,0)\right)$. The geometric validity of this reduction resides in the motion of slow manifold (fixed point), and as we have shown, the reduced zerodimensional model will be valid as long as the motion of the fixed point remains negligible (see FiguRES $3 \mathrm{a}$ and $3 \mathrm{~b}$ ).

As a final remark, we note, as a result of the scaling analysis, that the dynamics of $s_{2}$ (during its accumulation to $s_{2}^{\max }$ ) is expressible in terms of a 
Lambert-W function (when the indicator reaction is fast, see [5] for details),

$$
s_{2}=s_{2}^{\max }\left(1+\varpi W\left[-\frac{1}{\varpi} \exp \left(-\frac{1}{\varpi}-\frac{\left(s_{1}^{0} / t_{s_{1}}-V_{2}\right)^{2}}{V_{2} K_{M_{2}}} t\right)\right]\right)
$$

where $\varpi \equiv V_{2} / V_{1} \cdot\left(1+\sigma_{1}\right) / \sigma_{1}$. The natural timescale that arises from this expression is,

$$
t_{s_{2}}^{c}=\frac{K_{M_{2}}+s_{2}^{\max }}{k_{4} e_{2}^{0}}
$$

which is essentially characteristic of the time it takes for $s_{2}$ to accumulate $s_{2}^{\max }$ when the indicator reaction is fast. In this case, it is straightforward to show that

$$
s_{2}^{\max } \approx \frac{K_{M_{2}}}{\varpi-1} .
$$

If we rescale the mass action equations with respect to $\mathbb{T}=t / t_{s_{2}}^{c}$, then we obtain,

$$
\lambda^{\min } \frac{d \bar{c}_{2}}{d \mathbb{T}}=\left(1+\kappa_{2}\right)\left(1+\tilde{\sigma}_{2}\right)\left[\left(1-\frac{\tilde{\sigma}_{2}}{1+\tilde{\sigma}_{2}} \bar{c}_{2}\right) \bar{s}_{2}-\frac{1}{1+\tilde{\sigma}_{2}} \bar{c}_{2}\right]
$$

where $\lambda^{\mathrm{min}}$ is given by,

$$
\lambda^{\min }=\frac{e_{2}^{0}}{K_{M_{2}}+s_{2}^{\max }} .
$$

If $\lambda^{\min } \ll 1$, then the approach to $s_{2}^{\max }$ will occur along the slow manifold in the phase-plane. However, if $\lambda^{\mathrm{min}}$ is order unity, then the trajectory will move very rapidly until it catches the sliding fixed point, at which time the indicator reaction will remain in a QSS. Thus, if $\lambda^{\min }$ is large enough, the "fast transient" can be interpreted geometrically as the rapid approach to the fixed point in the phase-plane.

\subsubsection{Analysis of slow indicator reactions}

The indicator reaction will be slow in comparison to the non-observable reaction if $t_{s_{2}} \gg t_{s_{1}}$; thus, in the slow regime, we take $\delta_{S} \gg 1$. 


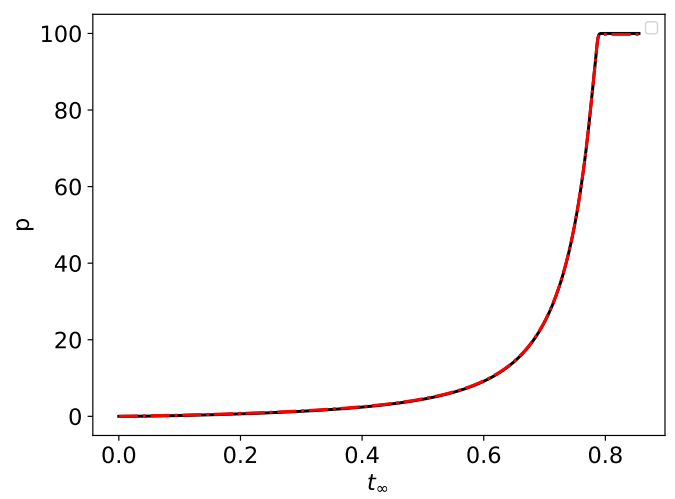

(a)

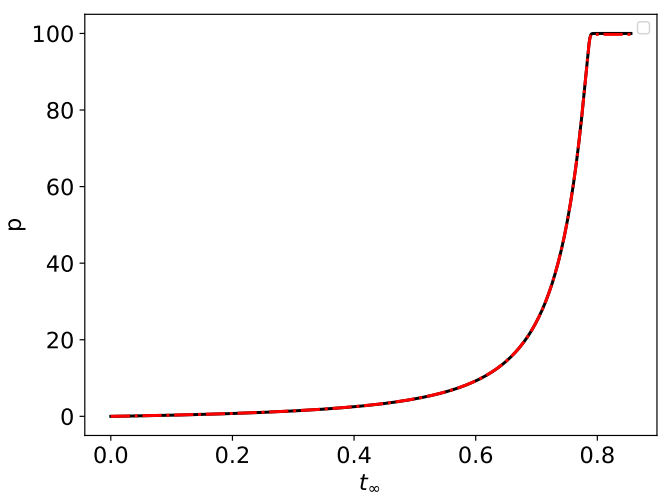

(b)

Figure 3: Time course of the product formation for the coupled sequential reaction mechanism when the indicator reaction is extremely fast. The solid black curve is the numerical solution to the mass action equation (13), $\dot{p}=k_{4} c_{2}$, and the broken red curve is the numerical solution to $\dot{p}=V_{1} s_{1} /\left(K_{M_{1}}+s_{1}\right)$. As shown, fast indicator reactions can be projected onto a zero-dimensional manifold, yielding a progress curve from which $K_{M_{1}}$ and $V_{1}$ can be estimated directly. The initial conditions and parameter values are: $e_{1}^{0}=1, s_{1}^{0}=100, k_{1}=1, k_{2}=1$ and $k_{-1}=1 . s_{2}^{0}=0, k_{3}=10, k_{4}=100$ and $k_{-3}=1$. In (a), $e_{2}^{0}=1$, and in (b) $e_{2}^{0}=1000$.

Since $t_{s_{1}}$ is now fast relative to $t_{s_{2}}$, we want to rescale the indicator reaction mass action equations with respect to $T$ :

$$
\begin{aligned}
\frac{d \bar{s}_{2}}{d T} & =\frac{\left(1+\sigma_{2}\right)\left(1+\kappa_{2}\right)}{\delta_{S}}\left[\left(\frac{\sigma_{2}}{1+\sigma_{2}} \bar{c}_{2}-1\right) \bar{s}_{2}+\frac{\alpha_{2}}{1+\sigma_{2}} \bar{c}_{2}\right]+\bar{c}_{1} \\
\lambda \frac{d \bar{c}_{2}}{d T} & =\frac{\left(1+\sigma_{2}\right)\left(1+\kappa_{2}\right)}{\delta_{S}}\left[\left(1-\frac{\sigma_{2}}{1+\sigma_{2}} \bar{c}_{2}\right) \bar{s}_{2}-\frac{1}{1+\sigma_{2}} \bar{c}_{2}\right]
\end{aligned}
$$

Looking carefully at the scaled equations, we see that if $\delta_{S} \gg\left(1+\sigma_{2}\right)\left(1+\kappa_{2}\right)$ then

$$
d s_{2}=-d s_{1}+\mathcal{O}\left(\delta_{S}\right)+\mathcal{O}(\varepsilon), \quad t_{c_{1}} \leq t \leq t_{s_{1}},
$$

which indicates the scaling of $\dot{s}_{2}$ is $\mathcal{O}(1)$ over the $t_{s_{1}}$ timescale. In order to reduce the equations via slow manifold projection over the $t_{s_{1}}$ timescale, it is thus necessary that

$$
\lambda \delta_{S} \ll\left(1+\sigma_{2}\right)\left(1+\kappa_{2}\right),
$$


which is equivalent to demanding adequate separation of the fast timescale $t_{c_{2}}$ and the depletion timescale $t_{s_{1}}$ :

$$
\lambda \delta_{S} \ll\left(1+\sigma_{2}\right)\left(1+\kappa_{2}\right) \equiv \frac{t_{c_{2}}}{t_{s_{1}}} \ll 1 .
$$

Given that the typical experimental initial conditions begin on the $c_{2}$-nullcline, two questions naturally arise from this analysis: (1) How does the slow manifold, $\mathcal{M}_{\lambda}$, emerge in the vector field when the QSS cannot be imposed when $t<t_{s_{1}}$ ? (2) What role does the fast timescale, $t_{c_{2}}$ play in determining the applicability of the QSSA?

First, we will analyze the higher order terms in the approximation to $\mathcal{M}_{\lambda}$. Since the sequential enzyme reaction can be analyzed in four-dimensional phase-space, we know that on the slow manifold

$$
c_{2}=h\left(s_{1}, c_{1}, s_{2}\right) \text {. }
$$

where $h: \mathbb{R}^{3} \rightarrow \mathbb{R}^{1}$ is an unknown function. Second, since $\mathcal{M}_{\lambda}$ is invariant, $h$ must satisfy the partial differential equation

$$
k_{3}\left(e_{2}^{0}-h\right) s_{2}-\left(k_{-3}+k_{4}\right) h=\boldsymbol{v} \cdot \nabla(h),
$$

where the differential operator $\nabla()$ is defined $\mathrm{as}^{2}$

$$
\nabla=\boldsymbol{i} \frac{\partial()}{\partial s_{1}}+\boldsymbol{j} \frac{\partial()}{\partial c_{1}}+\boldsymbol{k} \frac{\partial()}{\partial s_{2}}
$$

The velocity, $\boldsymbol{v}$, is given by:

$$
\boldsymbol{v}=\left[\dot{s}_{1}, \dot{c}_{1}, \dot{s}_{2}\right]^{T}
$$

We now want to approximate the solution to the dimensionless form of (50). To write the dimensionless form of (50), we rescale the concentrations of complex $\left(c_{1} \mapsto \bar{c}_{1}, c_{2} \mapsto \bar{c}_{2}\right)$ and substrate $\left(s_{1} \mapsto \bar{s}_{1}, s_{2} \mapsto \bar{s}_{2}\right)$ and introduce the dimensionless operator $\bar{\nabla}$ :

$$
\bar{\nabla}=\boldsymbol{i} \frac{\partial()}{\partial \bar{s}_{1}}+\boldsymbol{j} \frac{\partial()}{\partial \bar{c}_{1}}+\boldsymbol{k} \frac{\partial()}{\partial \bar{s}_{2}}
$$

\footnotetext{
${ }^{2}$ The vectors $\boldsymbol{i}, \boldsymbol{j}$ and $\boldsymbol{k}$ are the standard unit vectors in $\mathbb{R}^{3}$.
} 
Next, we are free to non-dimensionalize time with respect to any timescale. We choose $t_{s_{1}}$, and write the dimensionless velocity, $\overline{\boldsymbol{v}}$ as

$$
\overline{\boldsymbol{v}}=\left[\bar{s}_{1}^{\prime}, \bar{c}_{1}^{\prime}, \bar{s}_{2}^{\prime}\right]^{T}
$$

where prime denotes differentiation with respect $T=t / t_{s_{1}}$. The dimensionless form of (50) is then given by

$$
\frac{1}{\lambda} \frac{t_{s_{1}}}{t_{s_{2}}}\left[\left(1-\frac{\sigma_{2}}{1+\sigma_{2}} \bar{h}\right) \bar{s}_{2}-\frac{1}{1+\sigma_{2}} \bar{h}\right]=\overline{\boldsymbol{v}} \cdot \bar{\nabla}(\bar{h}) .
$$

To approximate the solution to (55), we will expand $\bar{h}$ in terms of a power series in $\lambda$

$$
\bar{h}=\sum_{i=0}^{\infty} \bar{h}_{i} \lambda^{i}
$$

where the $\bar{h}_{i}$ 's are functions of the dimensionless concentrations $\bar{s}_{1}, \bar{c}_{1}$ and $\bar{s}_{2}$ :

$$
\bar{h}_{i}=\bar{h}_{i}\left(\bar{s}_{1}, \bar{c}_{1}, \bar{s}_{2}\right) .
$$

Inserting (56) into (55) and collecting like terms yields, at zeroth order,

$$
\bar{h}_{0}=\frac{\left(\sigma_{2}+1\right)}{\sigma_{2} \bar{s}_{2}+1} \bar{s}_{2}
$$

which is identically the $\bar{c}_{2}$-nullcline. Additionally, at first order, we have,

$$
\bar{h}_{1}=\frac{\left(\sigma_{2}+1\right)^{2}}{\left(\sigma_{2} \bar{s}_{2}+1\right)^{4}\left(\kappa_{2}+1\right)} \bar{s}_{2}-\frac{\delta_{S}\left(\sigma_{2}+1\right)^{2}}{\left(\bar{s}_{2} \sigma_{2}+1\right)^{3}\left(\kappa_{2}\left(\sigma_{2}+1\right)+1\right)} \bar{c}_{1} .
$$

The second term on the right hand side of (59) represents (at first order) the time-dependency of the manifold $\mathcal{M}_{\lambda}$. We will denote this term as $\bar{h}_{1}(t)$ :

$$
\tilde{h}_{1} \equiv \frac{\left(\sigma_{2}+1\right)^{2}}{\left(\sigma_{2} \bar{s}_{2}+1\right)^{4}\left(\kappa_{2}+1\right)} \bar{s}_{2}, \quad \bar{h}_{1}(t) \equiv \frac{\delta_{S}\left(\sigma_{2}+1\right)^{2}}{\left(\bar{s}_{2} \sigma_{2}+1\right)^{3}\left(\kappa_{2}\left(\sigma_{2}+1\right)+1\right)} \bar{c}_{1} .
$$

Thus, up to first order, we have, as an approximation to the dynamics on $\mathcal{M}_{\lambda}$

$$
\bar{c}_{2}=\bar{h}_{0}+\lambda\left[\tilde{h}_{1}-\bar{h}_{1}(t)\right]+\mathcal{O}\left(\lambda^{2}\right)
$$


and the first order solution (59) is therefore comprised of two terms: $\tilde{h}_{1}$ and $\bar{h}_{1}(t)$. The truncated approximation

$$
\bar{c}_{2}=\bar{h}_{0}+\lambda \bar{h}_{1}+\mathcal{O}\left(\lambda \delta_{S} \bar{c}_{1}\right)
$$

is the static approximation, which gets closer to the $\bar{c}_{2}$-nullcline as $\lambda \rightarrow$ 0 . The term $\bar{h}_{1}(t)$ is dynamic, and accounts for the non-autonomous timedependency of the slow manifold as the non-observable reaction transpires. By inspection of (60), it is clear that if

$$
\delta_{S} \lambda \sim 1
$$

then the asymptotic expansion of $\mathcal{M}_{\lambda}$ may no longer be uniform when $c_{1}$ is large. In other words, a QSSA may not be appropriate on the $t_{s_{1}}$ timescale, over which we can assume that $c_{1}$ will be near its maximum value. What is the nature then, of the fast timescale $t_{c_{2}}$, and what does it tell us about the QSSA for the indicator reaction? Let us rescale the mass action equations with respect to $\bar{T}=t / t_{c_{2}}$ to obtain:

$$
\begin{aligned}
& \frac{d \bar{s}_{2}}{d \bar{T}}=\lambda\left[\left(\frac{\sigma_{2}}{1+\sigma_{2}} \bar{c}_{2}-1\right) \bar{s}_{2}+\frac{\alpha_{2}}{1+\sigma_{2}} \bar{c}_{2}\right]+\frac{t_{c_{2}}}{t_{s_{1}}} \bar{c}_{1}, \\
& \frac{d \bar{c}_{2}}{d \bar{T}}=\left[\left(1-\frac{\sigma_{2}}{1+\sigma_{2}} \bar{c}_{2}\right) \bar{s}_{2}-\frac{1}{1+\sigma_{2}} \bar{c}_{2}\right] .
\end{aligned}
$$

If $\lambda \ll 1$, but $t_{c_{2}} / t_{s_{1}}$ is order unity, then the $t_{c_{2}}$ is a timescale over which the accumulation of $s_{2}$ is linear, i.e.

$$
\dot{s}_{2}=k_{2} c_{1}+\mathcal{O}(\lambda), \quad t \leq t_{c_{2}} .
$$

One the other hand, if $t_{c_{2}} / t_{s_{1}} \ll 1$, then $t_{c_{2}}$ defines a lag time, which is a timescale over which the indicator reaction is essentially stationary.

Next, consider let us consider that $\lambda<t_{c_{2}} / t_{s_{1}} \ll 1$. In this case, the leading order solution is still given by (65), and the fast timescale $t_{c_{2}}$ defines a narrow time interval over which the accumulation of both $s_{2}$ and $c_{2}$ is asymptotically linear. Note this is analogous to the timescale $t_{c_{1}}$ in the nonobservable reaction, over which the growth of $c_{1}$ is asymptotically linear and the depletion of $s_{1}$ is negligible if $\varepsilon \ll 1$. In the context of the indicator 
reaction, $t_{c_{2}}$ defines a short timescale over which the accumulation is linear but asymptotically negligible. Additionally, since

$$
\lambda \delta_{S} \sim \frac{t_{c_{2}}}{t_{s_{1}}}
$$

we see that minimizing $t_{c_{2}}$ acts to reduce the region of non-uniformity in the asymptotic expansion of $\mathcal{M}_{\lambda}$. We also note that if $k_{-3} \gg k_{4}$, then, practically speaking, we can assume a QSS at $t=0$, since the higher order term $\bar{h}_{1}(t)$ is inversely proportional to $\left(1+\kappa_{2}\right)$ (see FIGURE $4 \mathrm{~d}$ ).

We can conclude that, as a rule of thumb, the smaller the ratio $t_{c_{2}} / t_{s_{1}}$, the more readily the QSSA can be imposed on the $t_{s_{1}}$ timescale when the indicator reaction is slow. However, if $\lambda \ll t_{c_{2}} / t_{s_{1}} \lesssim 1$, then a QSSA cannot be immediately imposed on the dynamical model of the mass action equations; therefore a QSSA should not be applied for $t \lesssim t_{s_{1}}$. Thus, the lag timescale influences when the QSSA is valid, in much the same way $t_{c_{1}}$ determines the onset of validity of the MM equation in the non-observable reaction (see Figures $4 \mathrm{a}-4 \mathrm{~d})$.

From the perspective of the dynamics in the four-dimensional phasespace, when $\delta_{S} \gg 1$, the non-observable reaction occurs so quickly that the phase space trajectory, at approximately $t=t_{s_{1}}$, lies extremely close to the intersection of the $s_{2}-c_{2}$ plane and the hyperplane $s_{2}=s_{1}^{0}$. In other words, when $\delta_{S}$ is sufficiently large, the reactions essentially decouple temporally:

$$
\begin{aligned}
& \dot{s}_{2}=\frac{V_{1}}{K_{M_{1}}+s_{1}} s_{1}, \quad t_{c_{1}} \leq t \leq t_{s_{1}}, \\
& \dot{s}_{2}=-\frac{V_{2}}{K_{M_{2}}+s_{2}} s_{2}, \quad t_{s_{1}}<t .
\end{aligned}
$$

Thus, we naturally obtain an inner solution (67a), valid for $t \leq t_{s_{1}}$, and an outer solution (67b), valid for $t>t_{s_{1}}$ (see [5] for technical details regarding the precise asymptotic expansions).

\section{The auxiliary enzyme reaction mechanism}

We now turn our attention to the auxiliary enzyme reaction mechanism described by the chemical equations (3)-(4). In this assay, the product of the non-observable reaction is the indicator enzyme $E_{2}[4]$. Following the same format utilized in the analysis of the sequential enzyme reaction mechanism, 


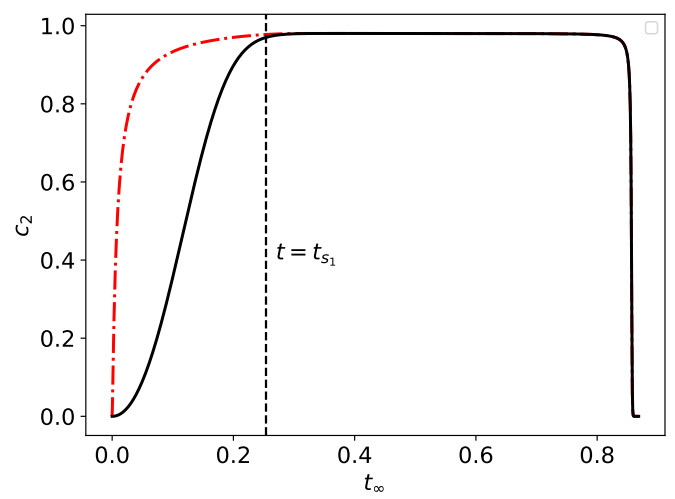

(a)

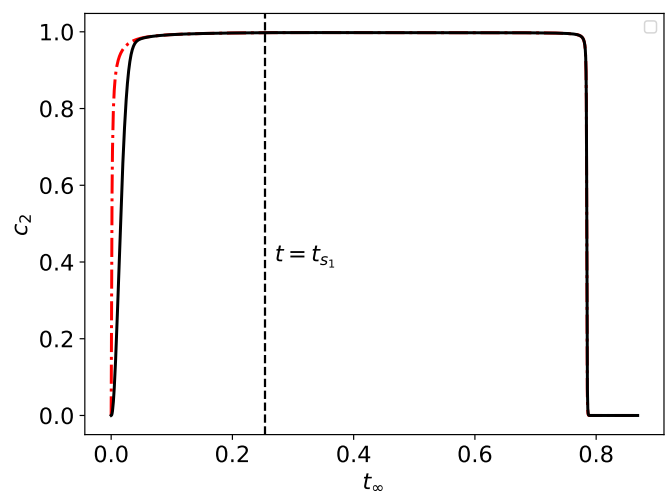

(c)

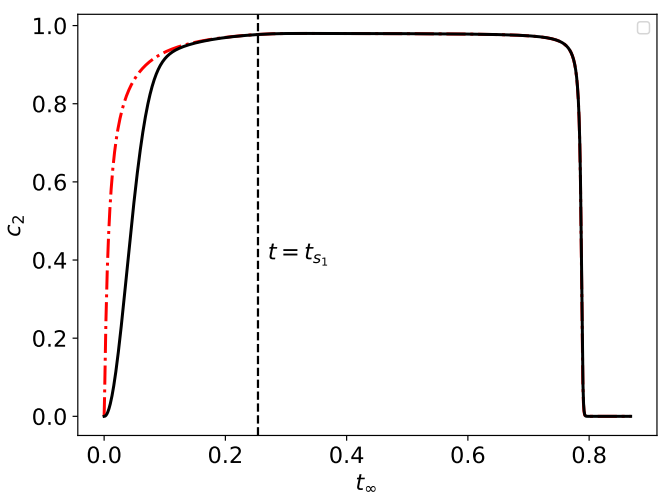

(b)

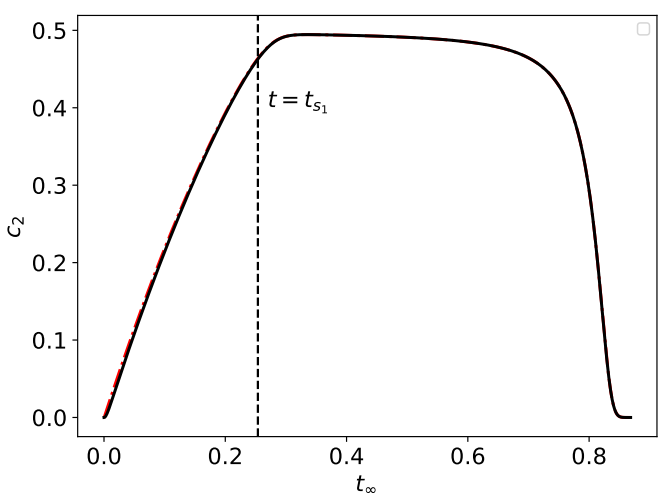

(d)

Figure 4: Numerical solution of the mass action equations (solid black curves) and the QSS approximation (27a) (broken red curve) for the coupled sequential enzyme reaction mechanism when the indicator reaction is slow. In all simulations $e_{1}^{0}=1, s_{1}^{0}=100, k_{1}=$ $10, k_{2}=100$ and $k_{-1}=1$ and $e_{2}^{0}=1$. Panel (a): $k_{3}=0.1, k_{-3}=0.1, k_{4}=0.1$. Panel (b): $k_{3}=1, k_{-3}=1, k_{4}=1$. Panel (c): $k_{3}=10, k_{-3}=1, k_{4}=1 . k_{3}=1, k_{-3}=100, k_{4}=1$. As the ratio $t_{c_{2}} / t_{s_{1}}$ gets smaller, the QSSA can be imposed almost immediately. In fact, if $k_{-3}$ is large, the QSSA can be imposed when $t=0$ as illustrated in panel (d). This is captured asymptotically by the fact that the order $\lambda$ terms in the expansion of $\mathcal{M}_{\lambda}$ are inversely proportional to $\left(1+\kappa_{2}\right)$.

we begin by scaling the mass action equations obtained by applying the law of mass action to (3)-(4). 


\subsection{Scaling of the auxiliary enzyme reaction}

The mass action equations that govern this reaction are:

$$
\begin{aligned}
& \dot{s}_{1}=-k_{1}\left(s_{1}^{0}-c_{1}\right) e_{1}+k_{-1} c_{1} \\
& \dot{c}_{1}=k_{1}\left(e_{1}^{0}-c_{1}\right) s_{1}-\left(k_{-1}+k_{2}\right) c_{1} \\
& \dot{s}_{2}=-k_{3}\left(e_{2}^{A}-c_{2}\right) s_{2}+k_{-3} c_{2} \\
& \dot{c}_{2}=k_{3}\left(e_{2}^{A}-c_{2}\right) s_{2}-\left(k_{-3}+k_{4}\right) c_{2},
\end{aligned}
$$

where $e_{2}^{A}$ denotes the concentration of the activated enzyme $E_{2}$ and is given by

$$
e_{2}^{A}=s_{1}^{0}-s_{1}-c_{1}
$$

with $s_{1}^{0}$ denoting the initial non-observable substrate $S_{1}$ concentration. Thus, the indicator reaction is described by a non-autonomous set of equations with $e_{2}^{A}(t)$ as its forcing term. As with the sequential enzyme reaction, the auxiliary enzyme reaction has four timescales. The timescales $t_{c_{1}}$ and $t_{s_{1}}$ are identical to those defined earlier in the sequential reaction. The additional timescales are $t_{c_{2}}^{a}$ and $t_{s_{2}}^{a}$

$$
t_{c_{2}}^{a}=\frac{1}{k_{3}\left(K_{M_{2}}+s_{2}^{0}\right)}, \quad t_{s_{2}}^{a}=\frac{K_{M_{2}}+s_{2}^{0}}{k_{4}\left\langle e_{2}^{A}\right\rangle},
$$

corresponding to the respective fast and slow timescales of the indicator reaction (see [4] for details regarding the validity of these timescales). The quantity $\left\langle e_{2}^{A}\right\rangle$ is the average amount of enzyme produced by the non-observable reaction over the duration of the indicator reaction. Rescaling the indicator reactions with respect to $t_{s_{1}}$ yields

$$
\begin{aligned}
\frac{d \tilde{s}_{2}}{d T} & =\frac{\max e_{2}^{A}}{\left\langle e_{2}^{A}\right\rangle} \frac{(1+\beta)\left(1+\kappa_{2}\right)}{\delta_{S}^{a}}\left[\left(\frac{\beta}{1+\beta} \tilde{c}_{2}-\tilde{e}_{2}^{A}\right) \tilde{s}_{2}+\frac{\alpha_{2}}{1+\beta} \tilde{c}_{2}\right] \\
\mu \frac{d \tilde{c}_{2}}{d T} & =\frac{\max e_{2}^{A}}{\left\langle e_{2}^{A}\right\rangle} \frac{(1+\beta)\left(1+\kappa_{2}\right)}{\delta_{S}^{a}}\left[\left(\tilde{e}_{2}^{A}-\frac{\beta}{1+\beta} \tilde{c}_{2}\right) \tilde{s}_{2}-\frac{1}{1+\beta} \tilde{c}_{2}\right],
\end{aligned}
$$

where $\mu, \beta, \delta_{S}^{a}, \tilde{s}_{2}, \max e_{2}^{A}, \tilde{e}_{2}^{A}$ and $\tilde{c}_{2}$ are given by:

$$
\begin{aligned}
& \delta_{S}^{a} \equiv t_{s_{2}}^{a} / t_{s_{1}}, \quad \beta \equiv \frac{s_{2}^{0}}{K_{M_{2}}}, \quad \mu \equiv \frac{\max e_{2}^{A}}{K_{M_{2}}+s_{2}^{0}}, \quad \tilde{e}_{2}^{A}=e_{2}^{A} / \max e_{2}^{A} \\
& \tilde{s}_{2} \equiv \frac{s_{2}}{s_{2}^{0}}, \quad \tilde{c}_{2} \equiv \frac{\left(K_{M_{2}}+s_{2}^{0}\right)}{s_{2}^{0} \max e_{2}^{A}} c_{2}, \quad \max e_{2}^{A} \equiv \max _{t \leq t_{s_{2}}^{a}} e_{2}^{A} .
\end{aligned}
$$


As in the case of the sequential enzyme reaction with $\lambda, \mu \ll 1$ is enough to establish the presence of a slow manifold. But, does the rate of the nonobservable reaction affect the validity of the leading order approximation

$$
c_{2}=\frac{e_{2}^{A} s_{2}}{K_{M_{2}}+s_{2}}+\mathcal{O}(\mu)
$$

If the indicator reaction is extremely fast, then we can assume that $\delta_{S}^{a} \ll$ 1. If $\mu \ll 1$, then the leading order approximation is

$$
c_{2}=\frac{e_{2}^{A}}{K_{M_{2}}+s_{2}} s_{2}+\mathcal{O}\left(\mu \delta_{S}^{a}\right)
$$




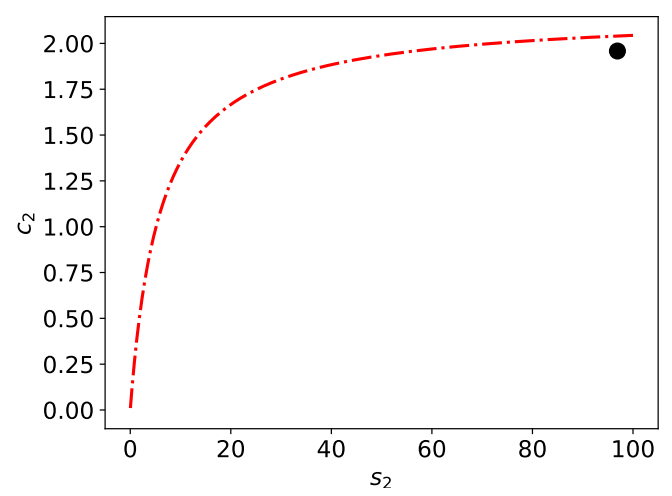

(a)

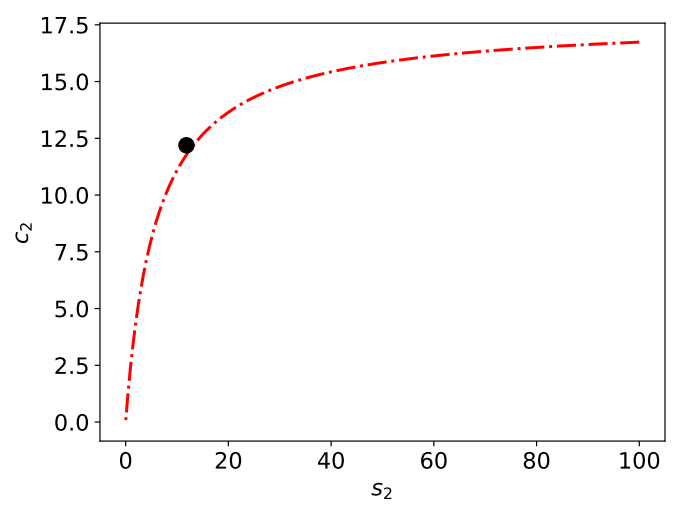

(c)

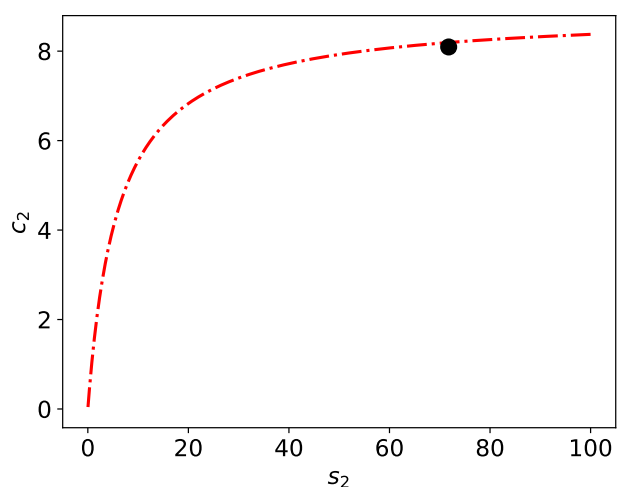

(b)

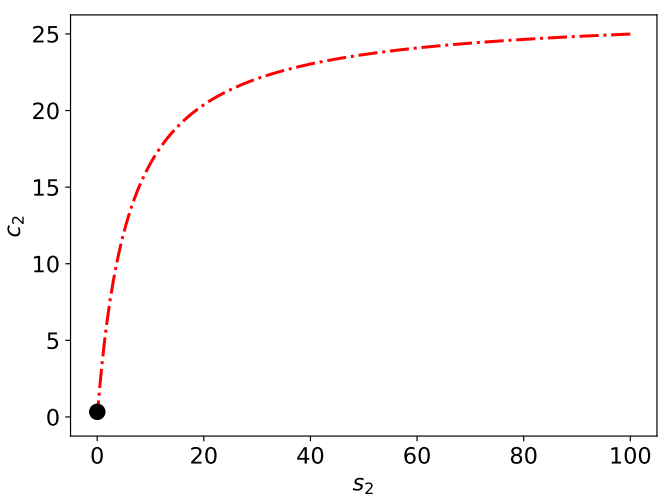

(d)

Figure 5: Snapshots of the numerical solution of the mass action equations (68) (solid black dot) and $c_{2}$-nullcline (broken red curve) for the coupled auxiliary enzyme reaction mechanism. This reaction mechanism exhibits a Laelaps manifold. Note that the numerical solution follows the $c_{2}$-nullcline, eventually catching it, and then descends towards the origin along a path that lies extremely close to the $c_{2}$-nullcline. The nullcline swings through the phase-plane almost like a windshield wiper that rotates counterclockwise. A dynamical representation of the Laelaps manifold is shown in Movie 2 (Supplementary Materials). The constants (without units) used in the numerical simulation are: $e_{1}^{0}=1, s_{1}^{0}=100, k_{1}=1, k_{2}=10$ and $k_{-1}=1 . s_{2}^{0}=100, k_{3}=1, k_{4}=5$ and $k_{-3}=1$.

and the depletion of substrate is given in terms of a Lambert-W function (again, see [4] for details regarding this particular solution):

$$
s_{2}=K_{M_{2}} W\left[\sigma_{2} \exp \left(\sigma_{2}-\frac{k_{4} \varpi t^{2}}{2 K_{M_{2}}}\right)\right], \quad \varpi \equiv \varepsilon k_{2} s_{1}^{0}
$$


Notice for fast indicator reactions, it is not necessary that $s_{2}^{0} \gg s_{1}^{0}$, as the amount of activated enzyme concentration $e_{2}$ produced by the non-observable will be small if the duration of the indicator reaction is short. Geometrically, the phase-plane solution catches the $c_{2}$-nullcline rather slowly; this is because the solution "hovers" under the $c_{2}$-nullcline for most of the reaction. It catches the $c_{2}$-nullcline towards the end of the reaction and then follows the $s_{2}$-nullcline and $c_{2}$-nullclines down to the origin of the phase-plane (see Figures 6a-6d).

Thus, if we observe the dynamics in the phase-plane, we see a long (although short relative to the depletion timescale of the non-observable reaction) period of depletion of $s_{2}$ and accumulation of $c_{2}$, followed by a small timescale over which the concentration $c_{2}$ rapidly diminishes (see FIGURE 7 ). Note that this is opposite of typical fast/slow systems, as there is usually a short timescale which accounts for rapid accumulation, followed by a long timescale the accounts for slow depletion. In the latter case, when the indicator reaction is extremely fast, we see slow/fast dynamics in the mass action equations of the indicator reaction; this is in contrast to the usual slow/fast dynamics of prototypical singularly perturbed equations.

\subsubsection{Analysis of slow indicator reactions}

As the indicator reaction begins to slow down, the slow depletion or accumulation timescale (present when the indicator reaction is fast) begins to shorten, and the timescale that accounts for the rapid depletion of $c_{2}$ begins to lengthen. As the speed of the indicator reaction begins to shorten, the dynamical behavior of the solution in the phase plane starts to resemble a more standard slow/fast problem (see FigURE 8).

The reason for this can be explained through scaling analysis. First, if the non-observable reaction is much faster than the indicator reaction, then we expect that

$$
\left\langle e_{2}^{A}\right\rangle=\lim _{t \rightarrow \infty} \frac{1}{t} \int_{0}^{t} e_{2}^{A} \mathrm{dt} \approx \mathrm{s}_{1}^{0} \equiv \sup _{0 \leq \mathrm{t}<\infty} \mathrm{e}_{2}^{\mathrm{A}}
$$

and thus we will take $T_{s_{2}}$

$$
T_{s_{2}}=\frac{K_{M_{2}}+s_{2}^{0}}{k_{4} s_{1}^{0}}
$$

to be the appropriate depletion timescale for the indicator reaction. Moreover, we will also assume that $\delta_{S}^{a} \gg 1$ for slow indicator reactions. Applying 


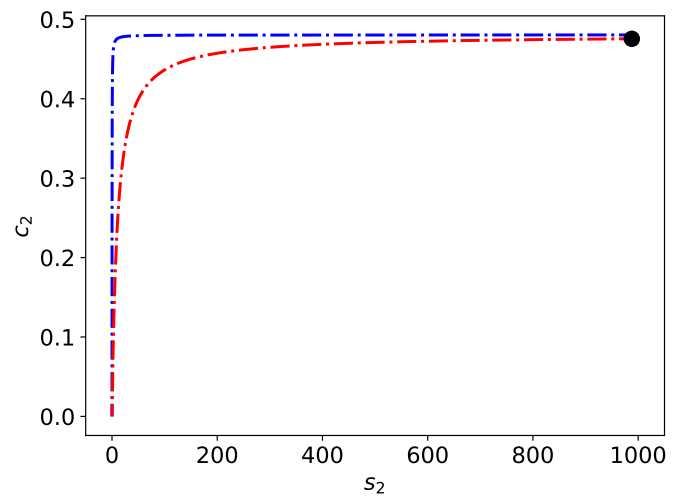

(a)

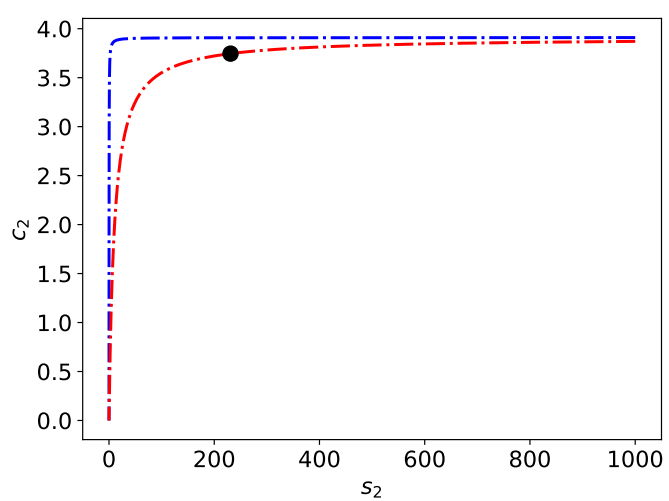

(c)

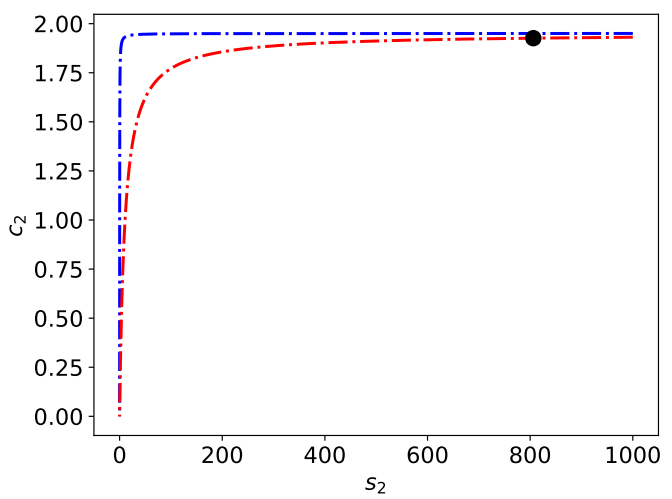

(b)

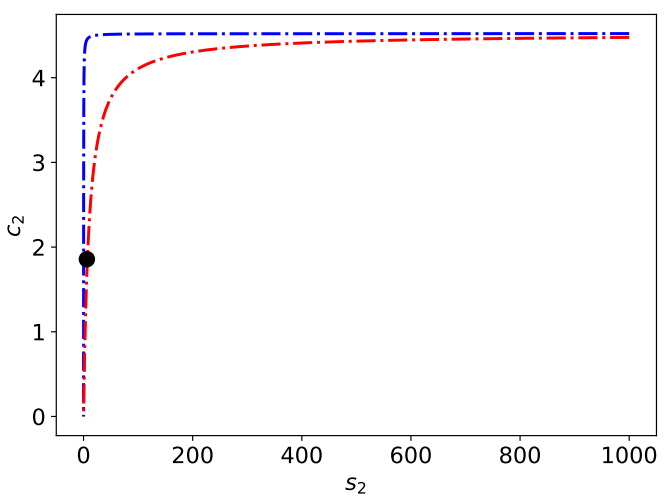

(d)

Figure 6: Snapshots of the numerical solution of the mass action equations (68) (solid black dot) and $c_{2}$ (broken red curve) and $s_{2}$ (broken blue curve) nullclines for the coupled auxiliary enzyme reaction mechanisms when the indicator reaction is fast. The constants (without units) used in the numerical simulation are: $e_{1}^{0}=1, s_{1}^{0}=100, k_{1}=1, k_{2}=1$ and $k_{-1}=1 . s_{2}^{0}=1000, k_{3}=10, k_{4}=100$ and $k_{-3}=1$.

the previous scaling laws, we obtain

$$
\begin{gathered}
\frac{d \tilde{s}_{2}}{d T}=\frac{(1+\beta)\left(1+\kappa_{2}\right)}{\delta_{S}^{a}}\left[\left(\frac{\beta}{1+\beta} \tilde{c}_{2}-\tilde{e}_{2}^{A}\right) \tilde{s}_{2}+\frac{\alpha_{2}}{1+\beta} \tilde{c}_{2}\right] \\
\mu \frac{d \tilde{c}_{2}}{d T}=\frac{(1+\beta)\left(1+\kappa_{2}\right)}{\delta_{S}^{a}}\left[\left(\tilde{e}_{2}^{A}-\frac{\beta}{1+\beta} \tilde{c}_{2}\right) \tilde{s}_{2}-\frac{1}{1+\beta} \tilde{c}_{2}\right],
\end{gathered}
$$




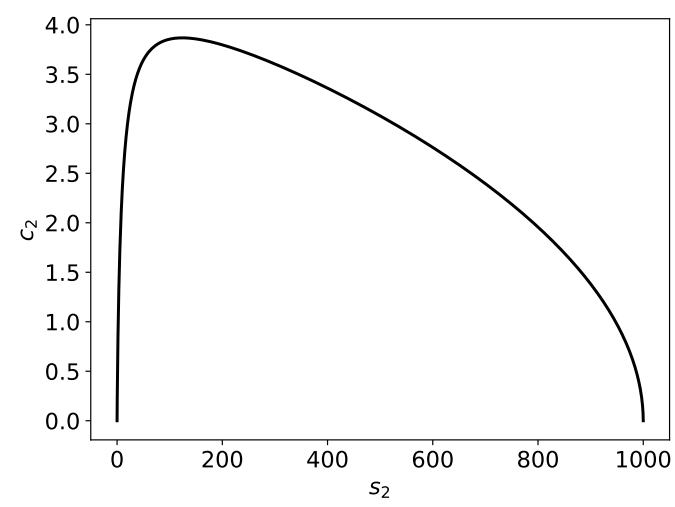

Figure 7: In the coupled enzyme auxiliary reaction mechanism when the indicator reaction is slow, phase-plane trajectories undergo a long period of rapid accumulation of $c_{2}$ followed by a short period of rapid depletion of $c_{2}$. In this simulation $k_{3}=1, k_{4}=100, k_{-3}=1, s_{2}^{0}=$ 1000 , and $k_{1}=1, k_{2}=1, k_{-1}=1, e_{1}^{0}=1$ and $s_{1}^{0}=100$.

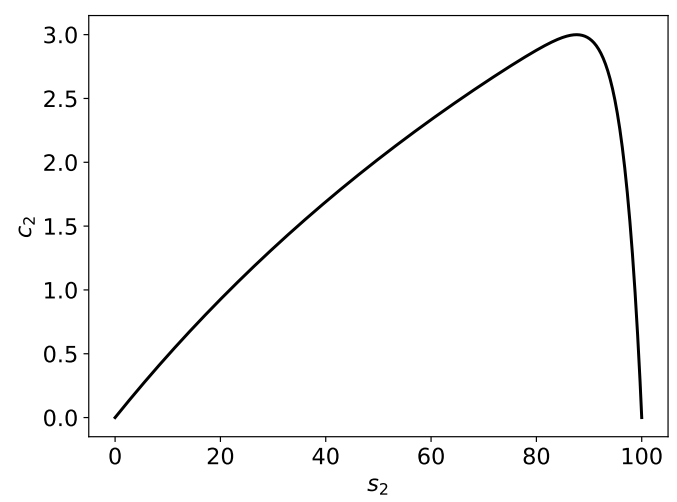

Figure 8: In the coupled auxiliary enzyme reaction mechanism when the indicator reaction is fast, phase-plane trajectories undergo a short period of rapid accumulation of $c_{2}$ followed by a longer period of slow depletion of $c_{2}$. In this simulation $k_{3}=1, k_{4}=1, k_{-3}=1, s_{2}^{0}=$ 100 , and $k_{1}=10, k_{2}=100, k_{-1}=1, e_{1}^{0}=1$ and $s_{1}^{0}=10$.

where $\mu$ and $\delta_{S}^{a}$ are now given by

$$
\mu \equiv \frac{s_{1}^{0}}{K_{M_{2}}+s_{2}^{0}}, \quad \delta_{S}^{a} \equiv \frac{T_{s_{2}}}{t_{s_{1}}} .
$$

The condition that $\mu \ll 1$ is necessary to ensure a QSSA on the depletion timescale (i.e., when $t>t_{s_{1}}$. However, by inspection, it is clear that if

$$
\delta_{S}^{a} \gg(1+\beta)\left(1+\kappa_{2}\right)
$$


then $s_{2}$ will be a slow variable for the duration of the non-observable reaction. The question we want to address is: Can we assume a QSSA for the duration of the non-observable reaction? First, notice we can define a RSA for the indicator reaction over $t_{s_{1}}$ as

$$
\max \left|\dot{s}_{2}\right| \cdot t_{s_{1}} \ll s_{2}^{0} \Longrightarrow \delta_{S}^{a} \gg(1+\beta)\left(1+\kappa_{2}\right),
$$

which is equivalent to the relationship we observe in the scaled equation (78a). On the other hand, if we demand that

$$
\mu \delta_{S}^{a} \ll(1+\beta)\left(1+\kappa_{2}\right),
$$

we find that it is necessary that

$$
\frac{t_{c_{2}}^{a}}{t_{s_{1}}} \ll 1
$$

where $t_{c_{2}}^{a}$ is given by

$$
t_{c_{2}}^{a} \equiv \frac{1}{k_{3}\left(K_{M_{2}}+s_{2}^{0}\right)} .
$$

The task now is to understand the significance of both (82) and (83), and the nature of $t_{c_{2}}$. To state things precisely, what happens when (80) holds but (82) does not? Can a QSSA for the indicator reaction still be employed for $t \geq 0$ ? To explore this relationship, we first rescale the mass action equations with respect to $\hat{t}=t / t_{c_{2}}^{a}$

$$
\begin{aligned}
& \frac{d \tilde{s}_{2}}{d \hat{t}}=\mu\left[\left(\frac{\beta}{1+\beta} \tilde{c}_{2}-\tilde{e}_{2}^{A}\right) \tilde{s}_{2}+\frac{\alpha_{2}}{1+\beta} \tilde{c}_{2}\right] \\
& \frac{d \tilde{c}_{2}}{d \hat{t}}=\left(\tilde{e}_{2}^{A}-\frac{\beta}{1+\beta} \tilde{c}_{2}\right) \tilde{s}_{2}-\frac{1}{1+\beta} \tilde{c}_{2},
\end{aligned}
$$

from which we see that $t_{c_{2}}^{a}$ defines a short time scale when $\mu \ll 1$. The takeaway is that if $t_{c_{2}}^{a}$ is very short, then we do not expect $s_{2}$ or $c_{2}$ to change very much along this timescale. Thus, $t_{c_{2}}^{a}$ is a lag time, similar to the timescale $t_{c_{2}}$ defined in the sequential reaction. However, if $t_{c_{2}}^{a}$ begins to lengthen, and approaches a magnitude that is comparable to $t_{s_{1}}$, then we expect $c_{2}$ to vary significantly over $t_{s_{1}}$. 
What happens, however, when $t_{c_{2}}$ is of the same order of magnitude of as $t_{s_{1}}$ ? In mathematical terms, this is

$$
\frac{1}{10} t_{s_{1}}<t_{c_{2}}^{a} \leq t_{s_{1}}
$$

Geometrically, the QSSA fails until $t$ is on the order of $t_{s_{2}}$. This is because in order to impose the QSSA, it is necessary that the equation for complex scale as

$$
\epsilon \dot{c}_{2}=f\left(c_{2}, s_{2}, t\right)
$$

where $\epsilon \ll 1$. If $\mu \delta_{S}^{a}$ is too large, and $t_{c_{2}}$ is similar in magnitude to $t_{s_{1}}$, then it is not asymptotically valid to approximate $c_{2}$ as being in a QSS during the time course of the non-observable reaction. Thus, in an analogous manner that was observed in the analysis of the sequential enzyme reaction, the timescale $t_{c_{2}}^{a}$ plays a critical role in regulating when the QSSA is valid (see Figures 9a and 9b).

Geometrically, the invalidity of the QSSA over the $t_{s_{1}}$ timescale is due to the fact that the $c_{2}$-nullcline propagates through the phase-plane at a speed that is initially much faster than the solution to the mass action equations (see FigurEs 10a and 10b for the geometrical interpretation).

As a concluding remark, we note that when the indicator reaction is extremely slow, we have

$$
\begin{aligned}
& s_{2}=s_{2}^{0}, \quad t \leq t_{s_{1}} \\
& s_{2}=K_{M_{2}} W\left[\beta \exp \left(\beta-\eta_{2} t\right)\right], \quad t \geq t_{s_{1}},
\end{aligned}
$$

where $\eta_{2} \equiv V_{2} / K_{M_{2}}$ and $W[\cdot]$ denotes the Lambert-W function. Thus, the reactions essentially decouple (temporally), and the indicator reaction resembles a single-enzyme, single-substrate reaction when $t \geq t_{s_{1}}$. Together, equations (88a) and (88b) constitute inner and outer solutions.

\section{Discussion}

In this work, two types of coupled enzyme reaction mechanisms - the sequential enzyme and auxiliary enzyme reaction mechanisms - assays have been analyzed through scaling analysis. The main contribution of this paper is the phase-plane analysis and geometric understanding of how scaling laws and how singularly perturbed problems in chemical kinetics can be analyzed 


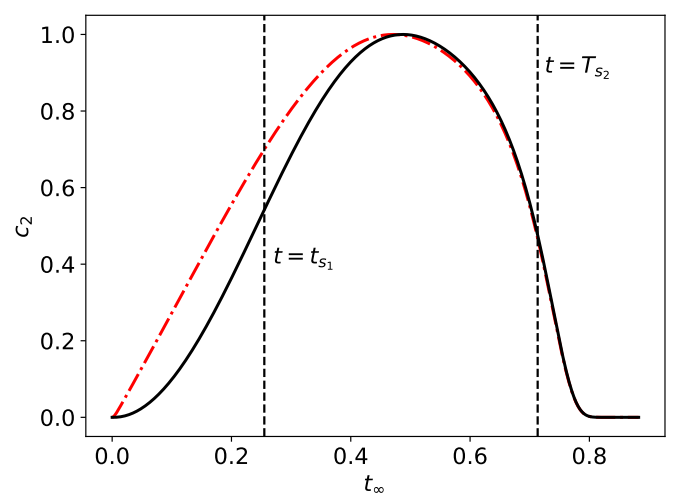

(a)

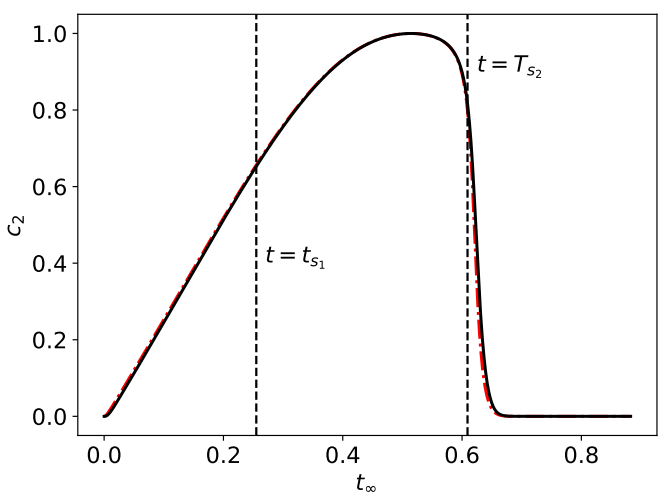

(b)

Figure 9: Illustration of the validity of the QSSA in the coupled auxiliary enzyme reaction mechanism. The broken red curve is the numerical solution corresponding to the QSS approximation (74), and the solid black curve is the numerical solution to the mass action equations (68). In panel (a), $\delta_{S}^{a} \approx 27, \mu \approx 0.03$ and $t_{c_{2}} / t_{s_{1}} \approx 0.3$. In panel (b), $\delta_{S}^{a} \approx 9.2$, $\mu \approx 0.098$ and $t_{c_{2}} / t_{s_{1}} \approx 0.0088$. Notice the QSSA approximation matches for all time in (b), even though $\mu$ is larger than in (a). This is because $t_{c_{2}} / t_{s_{1}}$ is very small, whereas in (a), $t_{c_{2}}$ is of the same order magnitude as $t_{s_{1}}$. (a) The constants (without units) used in the numerical simulation are: $e_{1}^{0}=1, s_{1}^{0}=10, k_{1}=1, k_{2}=100$ and $k_{-1}=1 . s_{2}^{0}=100$, $k_{3}=.01, k_{4}=1$ and $k_{-3}=1$. (b) The constants (without units) used in the numerical simulation are: $e_{1}^{0}=1, s_{1}^{0}=10, k_{1}=1, k_{2}=100$ and $k_{-1}=1 . s_{2}^{0}=100, k_{3}=1, k_{4}=1$ and $k_{-3}=1$. The concentration $c_{2}$ has been scaled by its maximum value, and time has been mapped to the $t_{\infty}$ scale: $t_{\infty}(t)=1-1 / \ln (t+e)$.

when multiple timescales - four timescales in our case - are part of the phase-plane dynamics.

From a mathematical perspective, we have shown that the mass action equations that model the indicator reactions of both the sequential and auxiliary enzyme reaction mechanisms are expressed in a general form as

$$
\begin{aligned}
\dot{s}_{2} & =\delta f\left(s_{2}, c_{2}, t\right) \\
\epsilon \dot{c}_{2} & =\delta g\left(s_{2}, c_{2}, t\right),
\end{aligned}
$$

where $\epsilon$ is proportional to the ratio of slow and fast timescales of the indicator reaction, and $\delta$ is the ratio of the slow timescales of the non-observable reaction to the indicator reaction.

In the case of the indicator reaction of the coupled sequential enzyme reaction mechanism we have shown that, if $\delta \gg 1$, then, the mass action 


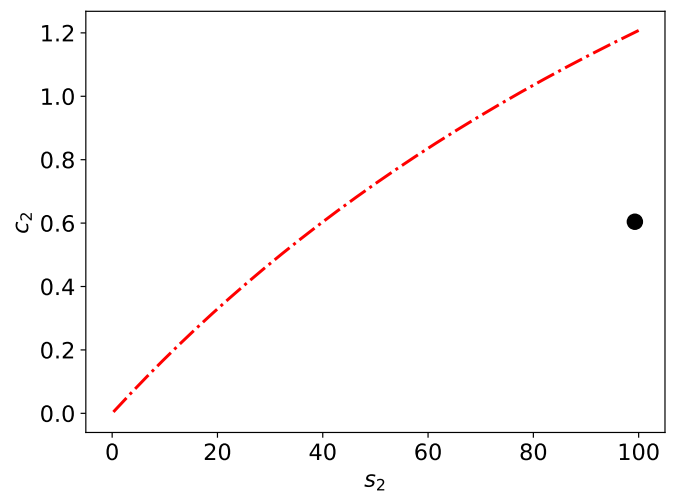

(a)

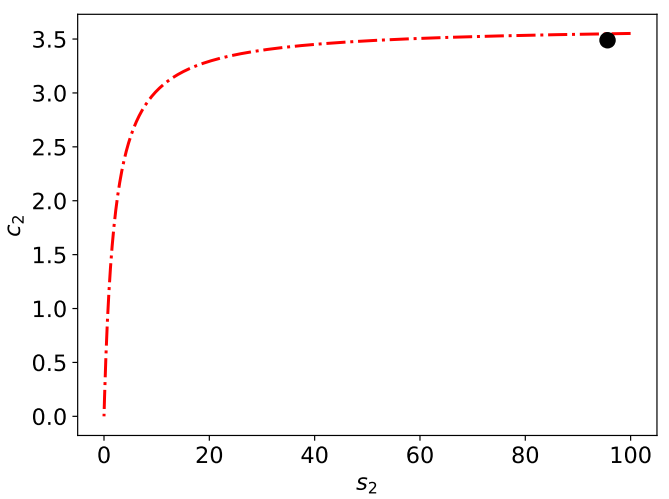

(b)

Figure 10: The geometric interpretation of the validity of the QSSA for the coupled auxiliary enzyme reaction mechanism. The broken red curve is a snapshot of the $c_{2}$ nullcline in the phase-plane, and the solid black dot is the corresponding snapshot of the numerical solution to the mass action equations (68). In panel (a), $\delta_{S}^{a} \approx 27, \mu \approx 0.03$ and $t_{c_{2}} / t_{s_{1}} \approx 0.3$. In panel $(\mathrm{b}), \delta_{S}^{a} \approx 9.2, \mu \approx 0.098$ and $t_{c_{2}} / t_{s_{1}} \approx 0.0088$. The numerical solution lags behind the $c_{2}$-nullcline in (a), whereas in (b), the solution "keeps up" with the swinging $c_{2}$-nullcline. The approximation gets better as $\mu$ and $t_{c_{2}} / t_{s_{1}}$ get smaller. (a) The constants (without units) used in the numerical simulation are: $e_{1}^{0}=1, s_{1}^{0}=10, k_{1}=$ $1, k_{2}=100$ and $k_{-1}=1 . s_{2}^{0}=100, k_{3}=.01, k_{4}=1$ and $k_{-3}=1$. (b) The constants (without units) used in the numerical simulation are: $e_{1}^{0}=1, s_{1}^{0}=10, k_{1}=1, k_{2}=100$ and $k_{-1}=1 . s_{2}^{0}=100, k_{3}=1, k_{4}=1$ and $k_{-3}=1$. Time has been mapped to the $t_{\infty}$ scale: $t_{\infty}(t)=1-1 / \ln (t+e)$.

equations can be can be approximated by a fixed point, $\boldsymbol{x}^{*}$. As $\delta \rightarrow \infty$, the approximation gets better and better. The significance of this result is imperative to the analysis of the inverse problem, when $K_{M_{1}}$ and $V_{1}$ are estimated by applying least linear or non-linear squares algorithms to experimental data collected from the indicator reaction. In the limit as $\delta \rightarrow \infty$, the rate expression for product reduces to

$$
\dot{p}=\frac{V_{1}}{K_{M_{1}}+s_{1}} s_{1},
$$

when the RSA holds for the non-observable reaction. Thus, $K_{M_{1}}$ and $V_{1}$ could be estimated by analyzing progress curves generated by the indicator reaction.

In contrast, we have shown that when $\delta \ll 1$, the non-observable and indicator reaction decouple temporally, in which case the mass action equations 
can effectively be analyzed separately as two, two-dimensional autonomous dynamical systems. Additionally, we have shown how fast timescales that arise naturally in the scaled mass action equations of the indicator reactions play a role in the determining the appropriate domain of validity for the QSSA. This result is novel, since initial conditions typically start on the critical manifold of the singularly perturbed equations and thus it would seem that a QSS could be imposed for all time. However, as we have shown, this assumption does not hold if the indicator reaction is slow and th lag timescale is too long. Moreover, if the indicator reaction is fast, then it must hold that:

$$
\lambda^{\min }=\frac{e_{2}^{0}}{K_{M_{2}}+s_{2}^{\max }} \ll 1, \quad \mu^{\max }=\frac{\max e_{2}^{A}}{K_{M_{2}}+s_{2}^{0}} \ll 1 .
$$

This result has been explained via scaling but also geometrically through phase plane analysis.

We hope that the applied mathematics and chemical kinetics communities will continue to investigate these types of reactions, as we feel there are still interesting and novel results to uncover.

\section{Acknowledgements}

This work is partially supported by the University of Michigan Protein Folding Diseases Initiative, and Beilstein-Institut zur Förderung der Chemischen Wissenschaften through its Beilstein Enzymology Symposia. We are grateful to Antonio Baici (University of Zurich) for helpful discussions about this work during the 2017 Beilstein Enzymology Symposia (Rüdesheim, Germany. WS is a fellow of the Michigan IRACDA program (NIH/NIGMS grant: K12 GM111725). We are grateful to Philip K. Maini (University of Oxford), Richard Bertram and Theo Vo (Florida State University) for their helpful comments on early drafts of this manuscript.

\section{References}

[1] S. Schnell, T. E. Turner, Reaction kinetics in intracellular environments with macromolecular crowding: simulations and rate laws, Prog. Biophys. Mol. Biol. 85 (2004) 235-260.

[2] D. L. Purich, Enzyme Kinetics: Catalysis \& Control. A Reference of Theory and Best-Practice Methods, Academic Press, London, UK, 2010. 
[3] F. B. Rudolph, B. W. Baugher, R. S. Beissner, Techniques in coupled enzyme assays, Methods Enzymol. 63 (1979) 22-42.

[4] J. Eilertsen, W. Stroberg, S. Schnell, Theory of the reactantstationary kinetics for a coupled enzyme assay, ChemRxiv (2018) DOI: 10.26434/chemrxiv.5631169.

[5] J. Eilertsen, S. Schnell, A kinetic analysis of coupled sequential enzyme reactions., ChemRxiv (2018) DOI: 10.26434/chemrxiv.5746065.

[6] L. Michaelis, M. L. Menten, Die Kinetik der Invertinwirkung, Biochem. Z. 49 (1913) 333-369.

[7] E. W. Davie, K. Fujikawa, W. Kisiel, The coagulation cascade: initiation, maintenance, and regulation, Biochemistry 30 (1991) 10363-10370.

[8] O. D Dang, A. Vindigni, E. Di Cera, An allosteric switch controls the procoagulant and anticoagulant activities of thrombin, Proceedings of the National Academy of Sciences of the United States of America 92 (1995) 5977-81.

[9] I. U. of Biochemistry, Symbolism and terminology in enzyme kinetics, Arch. Biochem. Biophys. 224 (1983) 732-740.

[10] A. Cornish-Bowden, Current IUBMB recommendations on enzyme nomenclature and kinetics, Perspect. Sci. 1 (2014) 74-87.

[11] F. E. Brot, M. L. Bender, Use of the specificity constant of .alpha.chymotrypsin, J. Am. Chem. Soc. 91 (1969) 7187-7191.

[12] D. E. Koshland, The application and usefulness of the ratio $k_{c a t} / k_{m}$, Bioorg. Chem. 30 (2002) 211-213.

[13] A. Cornish-Bowden, Fundamentals of enzyme kinetics, Wiley-Blackwell, Weinheim, Germany, 4th edition edition, 2012.

[14] A. Cornish-Bowden, Analysis of enzyme kinetics data, Oxford University Press, Oxford, 1995.

[15] W. Stroberg, S. Schnell, On the estimation errors of $K_{M}$ and V from time-course experiments using the Michaelis-Menten equation, Biophys. Chem. 219 (2016) 17-27. 
[16] W. Stroberg, S. Schnell, On the validity and errors of the pseudo-firstorder kinetics in ligandreceptor binding, Math. Biosci. 287 (2017) 3-11.

[17] W. R. McClure, Kinetic analysis of coupled enzyme assays, Biochemistry 8 (1969) 2782-2786.

[18] C. J. Barwell, B. Hess, The transient time of the hexokinase/pyruvate kinase/lactate dehydrogenase system in vitro, Hoppe-Seylers Z. Physiol. Chem. 351 (1970) 1531-1536.

[19] W. M. Hart, A kinetic model of a cyclic system for the fluorometric microdetermination of adenosine triphosphatase activity., Mol. Pharmacol. 6 (1970) 31-40.

[20] R. Goldman, E. Katchalski, Kinetic behavior of a two-enzyme membrane carrying out a consecutive set of reactions, J. Theor. Biol. 32 (1971) 243-257.

[21] J. S. Easterby, Coupled enzyme assays: A general expression for the transient, Biochim Biophys Acta. 293 (1973) 552-558.

[22] M. R. Roussel, S. J. Fraser, Geometry of the steady-state approximation: Perturbation and accelerated convergence methods, J. Chem. Phys. 93 (1990) 1072-1081.

[23] K. Albe, M. Butler, B. Wright, Cellular concentrations of enzymes and their substrates, J. theor. Biol. 143 (1990) 163-195.

[24] S. M. Hanson, S. Schnell, Reactant stationary approximation in enzyme kinetics, J. Phys. Chem. A 112 (2008) 8654-8658.

[25] S. Schnell, Validity of the Michaelis-Menten equation - Steady-state, or reactant stationary assumption: that is the question, FEBS J. 281 (2014) 464-472.

[26] C. Kuehn, Multiple time scale dynamics, volume 191 of Applied Mathematical Sciences, Springer-Verlag, New York, 2015.

[27] S. Wiggins, Introduction to applied nonlinear dynamical systems and chaos, volume 2 of Texts in Applied Mathematics, Springer-Verlag, New York, second edition, 2003. 
449

[28] O. K. Rice, Conditions for a steady state in chemical kinetics, J. Phys. Chem. 64 (1960) 1851-1857.

[29] S. K. Shoffner, S. Schnell, Approaches for the estimation of timescales in nonlinear dynamical systems: Timescale separation in enzyme kinetics as a case study, Math. Biosci. 287 (2017) 122-129.

[30] L. A. Segel, On the validity of the steady state assumption of enzyme kinetics, Bull. Math. Biol. 50 (1988) 579-593. 